\title{
LOCAL FUNCTIONS ON FINITE GROUPS
}

\author{
I. M. ISAACS AND GABRIEL NAVARRO
}

Abstract. We study local properties of finite groups using chains of $p$-subgroups.

\section{INTRODUCTION}

There are a number of character-counting conjectures for finite groups that suggest that certain global information is determined locally. For example, the famous McKay conjecture is of this type. (Recall that for a given prime $p$, and writing $m(X)$ to denote the number of irreducible characters with degree not divisible by $p$ for a finite group $X$, the McKay conjecture proposes that for an arbitrary finite group $G$, we have $m(G)=m(N)$, where $N$ is the normalizer of a Sylow $p$-subgroup of $G$.)

A fruitful approach to some of these local/global counting conjectures involves chains of $p$-subgroups. The first application of this technique to a charactercounting conjecture was in the paper [11] of R. Knörr and G. R. Robinson, where $p$-group chains arose fairly naturally to yield a reformulation of the Alperin weight conjecture. (The original statement of this conjecture appears in [1.) Additional connections between $p$-group chains and character counting appeared in papers by E. C. Dade, B. Külshammer, G. R. Robinson, J. Thévenaz, and others. (See, for example, 6], [12, [16, and [17.) Also, note that subgroup chains that are not necessarily $p$-group chains appear in [12.) It is not our intention here to give a complete history of the appearance of $p$-group chains in the literature, but we should mention early work of K. Brown and D. Quillen, where it seems that chains of subgroups, and specifically of $p$-subgroups, were first studied. (See [3], 4], and [15.)

In this partially expository paper, we adopt an entirely elementary point of view that enables us to recover results (or at least weaker, block-free forms of these results) of some of the aforementioned authors. More generally, we explore connections between $p$-group chains and the local/global counting of characters and other objects, and using our approach, we will see that in a surprisingly large number of cases, the various counts are determined locally. (To give one noncharacter example, we mention that given any $p$-group $J$, the number of conjugacy classes of subgroups of $G$ that are isomorphic to $J$ is locally determined.)

We should explain the phrase "block-free" in the previous paragraph. Character counts that involve a given prime $p$ often come in two varieties: block-free and

Received by the editors March 11, 2019, and, in revised form, November 18, 2019.

2010 Mathematics Subject Classification. Primary 20D99, 20D30, 20C15, 20D10.

This paper is partially based upon work supported by the NSF under grant DMS-1440140 while the authors were in residence at MSRI (Berkeley, CA), during the Spring 2018 semester. We thank the Institute for the hospitality and support.

The research of the second author was also supported by MTM2016-76196-P. 
block-by-block. For example, the McKay conjecture, as we stated it above, does not mention Brauer's p-blocks, and so we refer to it as "block-free", but a block-byblock refinement of the McKay conjecture due to Alperin is more precise: Instead of merely counting $p^{\prime}$-degree characters of a group $G$, the Alperin-McKay conjecture counts height zero characters in individual $p$-blocks of $G$. Usually, block-free versions of character-counting conjectures can easily be derived from their more general block-by-block formulations, and it is generally these simpler block-free statements that are amenable to our elementary methods, and which we discuss here.

We fix a prime $p$ for the remainder of this paper, and we recall that it is customary to say (somewhat vaguely) that information about a group $G$ is " $p$-local" or simply "local" if that information is somehow determined by considering only subgroups that are the normalizers in $G$ of nontrivial $p$-subgroups. In this paper, we use $p$-group chains in $G$ to give a precise definition that seems to capture remarkably well the informal notion of "local information". (Although our definition of "chainlocal" is new in a formal sense, connections between chains and local control have been known for some time, as, for example, in the Knörr-Robinson paper [11.)

We mention that using a different but related point of view, J. Thévenaz [17] has also given a precise definition of "local", for finite groups, and there is nontrivial intersection between his approach and ours. After we present our definition of "chain local" following Theorem [C, below, we offer for the sake of comparison, a brief description of Thévenaz's definition of "locally determined".

If $G$ is a finite group, a $p$-chain in $G$ is a collection $C=\left\{P_{i} \mid 0 \leq i \leq n\right\}$ of $p$-subgroups of $G$, where $1=P_{0}<P_{1}<\cdots<P_{n}$. We say that $n$ is the length of $C$, denoted len $(C)$, and we refer to the unique $p$-chain with length 0 as the trivial chain. (Since we are holding the prime $p$ fixed, we will usually refer to $p$-chains simply as "chains".)

A variant of this, which has been used by several authors, is based on the observation that since by our definition, the identity subgroup $P_{0}$ is a member of every chain, it contributes no information, and so it can be ignored. From that point of view (which we do not adopt), a $p$-chain would be a possibly empty totally ordered collection of nonidentity $p$-subgroups; its length would be its cardinality, and the trivial chain would be the empty chain.

We write $G_{C}$ to denote the stabilizer of the chain $C$ in the conjugation action of $G$ on the set of chains. Note that if $p$ divides $|G|$, then $p$ divides $\left|G_{C}\right|$ for all chains $C$. In fact, if $C$ is a nontrivial chain in $G$, then $G_{C}$ has a nonidentity normal $p$-subgroup, namely $\mathbf{Z}\left(P_{n}\right)$, where $n=\operatorname{len}(C)$.

Most of what follows will apply to arbitrary finite groups, but for a few of our results, it is convenient to be able to restrict attention to a smaller collection of groups, for example $p$-solvable groups. For this purpose we fix a family $\mathcal{F}$ of finite groups, with the property that if a group $G$ is isomorphic to a subgroup of some member of $\mathcal{F}$, then $G$ also lies in $\mathcal{F}$. We will refer to $\mathcal{F}$ as the "underlying family" of groups, but since almost all of our results will apply for an arbitrary underlying family, there will seldom be a need to refer to $\mathcal{F}$ explicitly. In the statements of the results in this paper, we will generally assume that a given group $G$ lies in the underlying family, and unless we specify otherwise, there is no loss in assuming that the underlying family consists of all finite groups. 
If $G$ is a finite group, we write $[G]$ to denote the isomorphism class of $G$, and we let $\mathbf{A}$ be the free-abelian group generated by the symbols $[G]$, where $G$ lies in the underlying family $\mathcal{F}$, and $|G|$ is divisible by $p$. We let $\mathbf{B}$ be the subgroup of $\mathbf{A}$ generated by all elements of the form

$$
B(G)=\sum_{C \in \mathcal{R}}(-1)^{\operatorname{len}(C)}\left[G_{C}\right],
$$

where $G$ runs over groups in the underlying family that have order divisible by $p$, and $\mathcal{R}$ is a set of representatives of the orbits of the conjugation action of $G$ on the set of chains in $G$. (Note that the element $B(G)$ of $\mathbf{A}$ defined by the above sum depends only on the isomorphism class of $G$, and it is independent of the choice of the representative set $\mathcal{R}$.)

In the literature, alternating $p$-chain sums (as, for example, in our definition of $B(G)$ above) have appeared using several different types of $p$-group chains. A somewhat surprising result (that we will not actually use in this paper) appears as Proposition 3.3 of [11, where Knörr and Robinson show that if instead of working with chains of arbitrary $p$-subgroups of $G$, we limit ourselves to chains of certain specific types of $p$-subgroups, this will not affect the values of the chain sums. We could, for example, work with chains of elementary abelian $p$-subgroups, as in Quillen's paper [15, or alternatively, we could use chains of radical $p$-subgroups. (Recall that a $p$-subgroup $Q$ of $G$ is said to be radical if $Q=\mathbf{O}_{p}\left(\mathbf{N}_{G}(Q)\right.$ ), and note that if we elect to work with radical chains, we require only that the nonidentity chain members $P_{i}$ with $i>0$ should be radical.) It follows, for example, that the element $B(G)$ of the free abelian group $\mathbf{A}$ is uniquely determined, regardless of which flavor of $p$-chain we decide to use, and so for simplicity, we always use chains of arbitrary $p$-subgroups in this paper. We have noticed, however, that for computer experiments that involve computations of chain sums, it seems that it is most efficient to work with chains of radical $p$-subgroups.

Next, we present a result that appears to be new.

Theorem A. Let $G$ be a finite group with order divisible by $p$. Then there exist uniquely determined (up to isomorphism) pairwise nonisomorphic finite groups $H_{i}$ and nonzero integers $n_{i}$ with $1 \leq i \leq r$, where $\mathbf{O}_{p}\left(H_{i}\right)>1$ for all $i$, and such that

$$
[G] \equiv n_{1}\left[H_{1}\right]+\cdots+n_{r}\left[H_{r}\right] \quad \bmod \mathbf{B} .
$$

Also, $\sum n_{i}=1$, and each of the groups $H_{i}$ is isomorphic to the stabilizer of some nontrivial chain in $G$.

Although each of the subgroups $H_{i}$ in Theorem $\mathrm{A}$ is isomorphic to some chain stabilizer $G_{C}$, where $C$ is a nontrivial $p$-chain of $G$, we do not know how to predict in advance, which chain stabilizers appear as the $H_{i}$.

The following are some examples (for the prime $p=2$ ) of congruences modulo $\mathbf{B}$, as in Theorem A

$$
\begin{aligned}
{\left[S_{5}\right] } & \equiv\left[S_{4}\right]+\left[D_{12}\right]-\left[C_{2} \times C_{2}\right] \\
{\left[S_{6}\right] } & \equiv 2\left[C_{2} \times S_{4}\right]-\left[C_{2} \times D_{8}\right] \\
{\left[\mathrm{SL}_{3}(2)\right] } & \equiv\left[A_{6}\right] \equiv 2\left[S_{4}\right]-\left[D_{8}\right] \\
{\left[M_{11}\right] } & \equiv\left[\mathrm{PSL}_{3}(3)\right] \equiv\left[S_{4}\right]+\left[\mathrm{GL}_{2}(3)\right]-\left[D_{8}\right], \\
{\left[A_{5}\right] } & \equiv\left[A_{4}\right]
\end{aligned}
$$


Also, some examples for the prime $p=3$ are:

$$
\begin{aligned}
{\left[\operatorname{PSL}_{3}(4)\right] } & \equiv\left[M_{10}\right] \equiv\left[\left(C_{3} \times C_{3}\right): Q_{8}\right], \\
{\left[A_{6}\right] } & \equiv\left[\left(C_{3} \times C_{3}\right): C_{4}\right], \\
{\left[S_{6}\right] } & \equiv\left[\left(C_{3} \times C_{3}\right): D_{8}\right], \\
\left.\operatorname{LSL}_{3}(3)\right] & \equiv 2\left[\left(C_{3} \times C_{3}\right): \mathrm{GL}_{2}(3)\right]-[N],
\end{aligned}
$$

where in the first example, $M_{10}$ is a point stabilizer in the Mathieu group $M_{11}$, and in the last example, $N$ is the normalizer of a Sylow 3 -subgroup in $\operatorname{PSL}_{3}(3)$.

Note that among the above examples, there are several pairs of groups $G$ and $H$ for which $[G] \equiv[H]$. We are able to describe precisely when this happens, and this defines a new equivalence relation on finite groups that we believe deserves further study.

Theorem B. Let $G$ and $H$ be finite groups with orders divisible by $p$. Then $[G] \equiv$ $[H] \bmod \mathbf{B}$ if and only if for every group $J$ such that $\mathbf{O}_{p}(J)>1$, the numbers of conjugacy classes of subgroups isomorphic to $J$ in $G$ and in $H$ are equal.

In the case where $H$ is a subgroup of $G$, there is a criterion that seems somewhat easier to understand.

Theorem C. Let $H \subseteq G$ be finite groups with order divisible by $p$. Then $[G] \equiv$ $[H] \bmod \mathbf{B}$, if and only if $\mathbf{N}_{G}(Q) \subseteq H$ for every nonidentity p-subgroup $Q$ of $H$.

Recall that a proper subgroup $H$ of $G$ is said to be strongly $p$-embedded in $G$ if $p$ divides $|H|$ but $p$ does not divide $\left|H \cap H^{g}\right|$ for elements $g \in G$ such that $g \notin H$. It is not hard to see that a proper subgroup $H$ of $G$ with order divisible by $p$ is strongly $p$-embedded in $G$ if and only if $H$ contains the full normalizer in $G$ of every nonidentity $p$-subgroup of $H$. (See Lemma 3.1, below.) The content of Theorem [C, therefore, is that if $H$ is a proper subgroup of $G$ with order divisible by $p$, then $[H] \equiv[G] \bmod \mathbf{B}$ if and only if $H$ is strongly $p$-embedded in $G$.

What is the connection, one might ask, between the group $\mathbf{A} / \mathbf{B}$ and global-local counting problems? Suppose that $f$ is an integer-valued function such that $f(G)$ is defined for all groups $G$ that lie in the underlying family and have order divisible by $p$, and assume that $f(G)=f(H)$ whenever $G \cong H$. (We say that such a function $f$ is isomorphism constant.) For example, $f(G)$ could be the number of irreducible characters of $G$ for which the $p$-part of the degree is exactly $p$, or the number of irreducible characters in the principal $p$-block of $G$, or the number of $p$-blocks of $G$. Some other examples that we will consider are the order of a Sylow $p$-subgroup of $G$, the number of conjugacy classes of $p$-singular elements of $G$ (i.e., elements that have order divisible by $p$ ) and the number of conjugacy classes of subgroups of $G$ that are isomorphic to some given group $J$, where $\mathbf{O}_{p}(J)>1$. Also, if the underlying family is $p$-solvable groups, we could take $f(G)$ to be the $p$-length of $G$.

In these examples, $f$ has values in $\mathbb{Z}$, but more generally, we consider isomorphism-constant functions with values in an arbitrary abelian group $U$. Observe that such a function defines a group homomorphism from $\mathbf{A}$ to $U$ by setting $f([G])=f(G)$, and we will often identify the function $f$ defined on groups with the corresponding homomorphism on the free-abelian group $\mathbf{A}$.

If $f$ is an isomorphism-constant function (or equivalently, $f$ is a homomorphism defined on $\mathbf{A}$ ), it may be possible to compute $f(G)$ using only local information about $G$, and in that case, it is customary to say that " $f$ is a local function". 
(For example, if $f(G)$ is the order of a Sylow $p$-subgroup of $G$, then $f$ is certainly local.) It is not completely clear, however, what information about a group should be considered to be local, nor is it always obvious for a given function $f$, whether or not $f(G)$ can be computed using only local information.

It is convenient, therefore, to have a precise definition of what it means for a function to be local. To avoid conflict with the existing definitions of "local", and since our definition relies on $p$-group chains, we shall say that a homomorphism defined on the free-abelian group $\mathbf{A}$ is chain local if the subgroup $\mathbf{B}$ of $\mathbf{A}$ is contained in $\operatorname{ker}(f)$. (To be more precise, we should say that " $f$ is chain local for $\mathcal{F}$ ", but generally we will not mention the underlying family $\mathcal{F}$.)

Equivalently, if $f$ is viewed as a function defined on groups, it is chain local precisely when

$$
\sum_{C \in \mathcal{R}}(-1)^{\operatorname{len}(C)} f\left(G_{C}\right)=0
$$

for all groups $G$ (in the underlying family) having order divisible by $p$, where as before, $\mathcal{R}$ is a set of representatives of the $G$-classes of chains of $p$-subgroups of $G$. A sum of the above form is independent of the choice of the representative set $\mathcal{R}$, and we refer to it as the chain sum on $G$ for the function $f$.

It seems appropriate to give a brief comparison of our definition of a chain local function and Thévenaz's definition of a locally determined function in 17 . We consider functions defined for all groups of order divisible by $p$, but Thévenaz considers functions $f$ defined on the poset of subgroups of a fixed group $G$, and he does not limit himself to subgroups having order divisible by $p$. He assumes that $f(H)=f(K)$ whenever $H$ and $K$ are conjugate in $G$, and he says that such a function $f$ is "locally determined" if the function $\hat{f}$, defined from $f$ via Möbius inversion vanishes on all subgroups $H$ such that $\mathbf{O}_{p}(H)=1$. Thévenaz then shows that his locally determined functions on the subgroups of a group $G$ are exactly the functions for which the chain sums vanish for all subgroups. Although these definitions are closely related, there are differences. For example, we shall see that the constant function $f(G)=1$ is chain local, but the function defined by $f(H)=1$ for every subgroup $H$ of $G$ is not locally determined in Thévenaz's sense.

Given a chain-local homomorphism $f$ defined on $\mathbf{A}$, it should be clear that if $[G] \equiv \sum n_{i}\left[H_{i}\right]$ as in Theorem $\mathrm{A}$, then $f(G)=\sum n_{i} f\left(H_{i}\right)$. If $f$ is chain local, therefore, then $f(G)$ is determined by the values of $f$ at the groups $H_{i}$, and thus Theorem $\mathrm{A}$ can be used as a tool to prove that certain functions are not chain local.

For example, suppose $p=2$ and $f(G)$ is the number of irreducible characters of $G$ whose degree has 2-part exactly equal to 2 . To see that $f$ fails to be chain local, we calculate $f$ on both sides of the expression

$$
\left[S_{5}\right] \equiv\left[S_{4}\right]+\left[D_{12}\right]-\left[C_{2} \times C_{2}\right],
$$

and we observe that we obtain unequal results. In fact, $f\left(S_{5}\right)=1$ while $f\left(S_{4}\right)+$ $f\left(D_{12}\right)-f\left(C_{2} \times C_{2}\right)=3$. We will show, however, that the function whose value at $G$ is the order of a Sylow $p$-subgroup is chain local, as is the number of conjugacy classes of $G$ that consist of $p$-singular elements. Also, it is a consequence of Dade's "ordinary conjecture" (see [6]) that the number of irreducible characters in the principal $p$-block of $G$ is chain local.

In order to link our definition of "chain-local" with the traditional notion of "local information", observe that the groups $H_{i}$ of Theorem $\mathrm{A}$ are isomorphic to 
subgroups of $G$, and each of them has a nonidentity normal $p$-subgroup. We can thus view $f\left(H_{i}\right)$ as local information for $G$, and so if $f$ is chain local, then since $f(G)$ is determined by the $f\left(H_{i}\right)$, we see that $f(G)$ can also be viewed as local information about $G$.

The chain local functions, therefore, are "local" in the traditional sense. Although perhaps it is somewhat less obvious, it is also true that conversely, if $f(G)=\sum n_{i} f\left(H_{i}\right)$ whenever $[G] \equiv \sum n_{i}\left[H_{i}\right]$ as in Theorem $\mathrm{A}$, then $f$ must be chain local.

Next, we discuss some further connections between character-counting conjectures and chain-local functions. For example, the following shows that the McKay conjecture is equivalent to the assertion that the McKay function $m$ is chain local, where $m(G)$ is the number of irreducible characters of a group $G$ that have degree not divisible by $p$.

Theorem D. The McKay function $m$ is chain local if and only if for every finite group $G$, we have $m(G)=m(N)$, where $N=\mathbf{N}_{G}(P)$ and $P \in \operatorname{Syl}_{p}(G)$.

We recall now some fairly standard notation. The function $k(G)$ is the number of irreducible characters of $G$ and $l(G)$ is the number of irreducible $p$-Brauer characters of $G$. (Of course, $k$ and $l$ also count the number of classes of $G$ and the number of classes of $p$-regular elements of $G$, respectively.) For integers $d \geq 0$, we write $k_{d}(G)$ to denote the number of irreducible characters of $G$ that have defect $d$. (Recall that the defect of a character $\chi \in \operatorname{Irr}(G)$ is the number $d$ such that $p^{d}$ is the $p$-part of the integer $|G| / \chi(1)$.)

The Alperin weight conjecture in the Knörr-Robinson reformulation 11 asserts that the function $l-k_{0}$ is chain local. (This was also explored by J. Thévenaz in [17.) Also, it can be seen that Dade's ordinary conjecture in its block-free form, stated as Conjecture 9.25 in [14, implies that all of the functions $k_{d}$ for $d>0$ are chain local. Since sums and differences of chain-local functions are clearly chain local, Dade's conjecture implies that the function

$$
k-k_{0}=\sum_{d>0} k_{d}
$$

is chain local. Now Knörr and Robinson proved in 11 that the function $k-l$ is chain local, so assuming Dade's ordinary conjecture, we see that the function $l-k_{0}=\left(k-k_{0}\right)-(k-l)$ is chain local, and as we remarked, this is equivalent to the Alperin weight conjecture. This shows that in their block-free forms, Dade's ordinary conjecture implies the Alperin weight conjecture. (Actually, Dade proved a stronger result in [6], namely that in their full block-by-block generality, Alperin's conjecture follows from Dade's.)

Of course, the Knörr-Robinson observation in [1] that the function $k-l$ is chain local, is the key to the above proof that the block-free form of the Alperin weight conjecture is a consequence of the block-free form of Dade's ordinary conjecture. As we shall see in Theorem E(b), below, our methods yield an elementary and character-free proof that $k-l$ is chain local, but we stress that our result is considerably weaker than the theorem in [11]: Working with characters and Brauer characters, Knörr and Robinson obtained results for individual $p$-blocks. To be specific, they showed that for each block $B$, the function $k(B)-l(B)$ is locally 
determined, where $k(B)$ and $l(B)$ are, respectively, the numbers of irreducible ordinary and Brauer characters in $B$. (We mention that Knörr and Robinson claim that the fact that $k(B)-l(B)$ is locally determined was actually known to Brauer.)

We will also use our methods to give an elementary proof that the block-free form of Dade's ordinary conjecture implies the McKay conjecture. (Another proof of this fact appeared recently in [14.) We mention that Dade claims in [6] that his more comprehensive "projective" conjecture implies the full block-by-block AlperinMcKay conjecture, and more recently, it was shown by R. Kessar and M. Linckelmann [10] that in fact, Dade's ordinary conjecture is sufficent to prove the AlperinMcKay conjecture.

Are there other chain-local functions that might interest us? How can we build local functions? What other local (in the traditional sense) properties of a group are reflected in chain-local functions? In Theorems $\mathrm{E}$ and F, below, we address these questions. (As we have said, Theorem $\mathrm{E}(\mathrm{b})$ below, is a weak form of a result of Knörr and Robinson. In fact, several of the functions described in Theorem E are already known to be "local", at least to the experts, but we give proofs here that all of these functions are chain local in the sense of our formal definition.)

For Theorem E(i), we recall that by definition, the defect of a conjugacy class $K$ of a group $G$ is the integer $d$ such that $p^{d}$ is the full $p$-part of $|G| /|K|$.

Theorem E. The following specific integer-valued isomorphism-constant functions defined on finite groups having order divisible by $p$ are chain local:

(a) the function that counts the conjugacy classes of $G$ that consist of p-elements, including the identity;

(b) the function $k-l$, which counts the conjugacy classes of $G$ that consist of p-singular elements;

(c) the function that counts the p-blocks of $G$ that have positive defect;

(d) the function $k_{1}$, which counts the irreducible characters of $G$ that have defect 1 ;

(e) the function that counts the conjugacy classes of subgroups of $G$ that are isomorphic to some given group $J$, where $\mathbf{O}_{p}(J)>1$;

(f) the function that counts the conjugacy classes of nontrivial p-subgroups $Q$ of $G$ such that $\mathbf{N}_{G}(Q)$ is isomorphic to some given group $J$;

(g) the function that counts the conjugacy classes of nontrivial radical p-subgroups $Q$ of $G$ such that $\mathbf{N}_{G}(Q)$ is isomorphic to some given group $J$.

(h) The constant function $f(G)=1$;

(i) the function that counts the conjugacy classes of $G$ that have defect $d$, where $d>0$.

Theorem $\mathrm{F}$, below, describes some general conditions that guarantee that a function is chain local, and it provides a way to construct new chain-local functions. (For example, we use Theorem Fin Corollary 6.3 to construct a chain-local function that detects whether or not a group $G$ is $p$-nilpotent.) We show in Theorem G that this technique can, in principle, be used to construct all possible chain-local functions.

Theorem F. A function $f$ defined on finite groups having order divisible by $p$ is chain local if it satisfies one of the following conditions:

(a) $f$ is isomorphism constant and $f(G)=f(N)$ whenever $N$ is the normalizer of a Sylow p-subgroup of $G$; 
(b) $f(G)=\sum_{Q} h\left(\mathbf{N}_{G}(Q)\right)$ for some isomorphism-constant function $h$, where $Q$ runs over a set of representatives of the conjugacy classes of nonidentity p-subgroups of $G$;

(c) $f(G)=\sum_{Q} h\left(\mathbf{N}_{G}(Q)\right)$ for some isomorphism-constant function $h$, where $Q$ runs over a set of representatives of the conjugacy classes of nonidentity radical p-subgroups of $G$.

For Theorem $\mathbb{F}(a)$, we should not ignore the fact that our underlying family $\mathcal{F}$ may be smaller than "all groups". What this result actually says is that if $f$ is isomorphism constant and $f(G)=f(N)$ for all groups $G$ having order divisible by $p$ and lying in the underlying family $\mathcal{F}$, then $f$ is chain local for $\mathcal{F}$. There is no need to verify the hypothesis that $f(G)=f(N)$ for groups $G$ not lying in $\mathcal{F}$, and in fact, $f(G)$ may be undefined for such groups. For example, suppose that $\mathcal{F}$ is the family of $p$-solvable groups, and let $f(G)$ be the number of $p^{\prime}$-special characters of $G$. (See [9] for the definition of a $\pi$-special character of a $\pi$-separable group, where $\pi$ is an arbitrary set of primes.) It is a fact that $f(G)=f(N)$ for all $p$-solvable groups $G$, and that is sufficient for us to conclude that $f$ is chain local for $p$-solvable groups.

It follows by Theorem $\mathrm{F}(\mathrm{a})$ that a function $f$ is chain local if it is possible to compute $f(G)$ by looking only at the normalizer $N$ of a Sylow $p$-subgroup $P$ of $G$. For example, the function $f(G)=|N|$, has this property, and so it is chain local. For the same reason, we see that if $f$ is a constant function, then $f$ is chain local, as is the function $f(G)=|\operatorname{Irr}(P)|$. Also, observe that if the McKay conjecture is true, then Theorem $\mathrm{F}$ (a) guarantees that the McKay function $m$ is chain local, and this proves half of Theorem D.

By Theorem $\mathrm{F}(\mathrm{b})$, every function $f$ that is local (in the sense that $f(G)$ is determined by the normalizers of nontrivial $p$-subgroups of $G$ ) is guaranteed to be chain local. In fact, to prove that a function $f$ is chain local, it is sufficient by Theorem $\mathrm{F}$ (c) to check that $f$ is determined by the normalizers of the nontrivial radical $p$-subgroups of $G$.

We shall see that Theorem E(c) follows from Theorem F(c) by Brauer's first main theorem. Also, Theorem E(d), which is a special case of Dade's conjecture, follows from Theorem $\mathrm{F}(\mathrm{b})$, but its proof requires some deep results of Brauer and Dade.

Theorem F(c) also provides an alternative proof that the Alperin weight conjecture in its original form implies the Knörr-Robinson reformulation of the conjecture. To see this, observe that Alperin's original statement of his conjecture is essentially that

$$
\sum_{Q} k_{0}\left(\mathbf{N}_{G}(Q)\right)=l(G)-k_{0}(G),
$$

where the sum runs over a set of representatives of the conjugacy classes of nonidentity radical $p$-subgroups of $G$. Assuming this, it follows by Theorem $\mathbf{F}$ (c) that the function $l-k_{0}$ is chain local, and as we have seen, this is exactly the Knörr-Robinson reformulation of the Alperin weight conjecture.

The following shows that Theorems $\mathrm{F}(\mathrm{b})$ and $\mathrm{F}(\mathrm{c})$ provide machines that are powerful enough to construct essentially all chain-local functions.

Theorem G. Let $f$ be a chain-local function with values in an abelian group $U$. 
(a) If $U=\mathbb{Q}^{+}$, the additive group of the rational numbers, then there exists a unique $U$-valued isomorphism-constant function $h$ defined on groups $N$ with $\mathbf{O}_{p}(N)>1$ and such that if $p$ divides $|G|$, then $f(G)=\sum_{Q} h\left(\mathbf{N}_{G}(Q)\right)$, where $Q$ runs over a set of representatives of the conjugacy classes of nonidentity $p$-subgroups of $G$.

(b) With no extra assumption on $U$, there exists a unique $U$-valued isomorphismconstant function $h$ defined on groups $N$ with $\mathbf{O}_{p}(N)>1$ and such that if $p$ divides $|G|$, then $f(G)=\sum_{Q} h\left(\mathbf{N}_{G}(Q)\right)$, where $Q$ runs over a set of representatives of the conjugacy classes of nonidentity radical p-subgroups of $G$.

Note that the requirement in Theorem G(a) that $f$ must be rational valued does not preclude the possibility that $f$ is actually integer valued. In that case, however, the unique function $h$ whose existence is guaranteed by the theorem may not be integer valued, although of course, it is rational valued.

We return now to the McKay conjecture. If the conjecture is true, then as we have seen, the McKay function $m$ is chain local, but in fact, even more is true: $m$ is "strongly" chain local. To explain this, consider an isomorphism-constant function $f$ with values in some abelian group $U$. Given $u \in U$, we can define a new, integervalued isomorphism-constant function $f_{(u)}$ by setting $f_{(u)}(G)=1$ if $f(G)=u$ and $f_{(u)}(G)=0$ otherwise. Clearly,

$$
f(G)=\sum_{u \in U} f_{(u)}(G) u
$$

so if $f_{(u)}$ is chain local for every element $u \in U$, it follows that $f$ is chain local. In this case, we say that $f$ is strongly chain local.

Note that if $f$ is an arbitrary chain local function with values in an abelian group $U$, and if $h$ is a function from $U$ into an abelian group $V$, then the composite function $h(f(G))$ is chain local whenever $h$ is a homomorphism. If $f$ is strongly chain local, however, then in fact, the composite function $h(f(G))$ is chain local for every choice of the function $h: U \rightarrow V$, whether or not $h$ is a homomorphism. Thus, for example, if $f$ is an integer-valued strongly chain-local function, then the function $f(G)^{2}$ is also chain local.

To see that $h(f(G))$ is chain local if $f$ is strongly chain local, let $u \in U$, and observe that since $f_{(u)}$ is chain local, we have

$$
0=\sum_{C \in \mathcal{R}}(-1)^{\operatorname{len}(C)} f_{(u)}\left(G_{C}\right)=\sum_{C \in \mathcal{R}_{u}}(-1)^{\operatorname{len}(C)},
$$

where $\mathcal{R}_{u}=\left\{C \in \mathcal{R} \mid f\left(G_{C}\right)=u\right\}$. Since $\mathcal{R}$ is partitioned by the subsets $\mathcal{R}_{u}$ for $u \in U$, we have

$$
\sum_{C \in \mathcal{R}}(-1)^{\operatorname{len}(C)} h\left(f\left(G_{C}\right)\right)=\sum_{u \in U} h(u) \sum_{C \in \mathcal{R}_{u}}(-1)^{\operatorname{len}(C)}=0,
$$

so the function $h(f(G))$ is chain local, as claimed.

Although the functions $k_{d}$ for $d>0$ are expected to be chain local by Dade's conjecture, experiment shows that these functions are not generally strongly chain local. For example, taking $p=2$, one can compute that

$$
\left[S_{3} \times S_{3}\right] \equiv 2\left[D_{12}\right]-\left[C_{2} \times C_{2}\right] \bmod \mathbf{B} .
$$


Now $k_{1}\left(S_{3} \times S_{3}\right)=4$, and we see that $k_{1}\left(D_{12}\right)=2$ and $k_{1}\left(C_{2} \times C_{2}\right)=0$, so we have

$$
k_{1}\left(S_{3} \times S_{3}\right)=2 k_{1}\left(D_{12}\right)-k_{1}\left(C_{2} \times C_{2}\right),
$$

as expected. The corresponding equality fails, however, if we replace $k_{1}$ by the function $\left(k_{1}\right)^{2}$, and we conclude that $k_{1}$ is not strongly chain local. In fact, most of the chain-local functions that we have discussed are not strongly chain local, but the McKay function appears to be an exception.

Theorem H. Assuming that the McKay conjecture is true, it follows that the McKay function $m$ is strongly chain local.

We shall see that if our underlying family $\mathcal{F}$ is the family of $p$-solvable groups, then there are many functions that are strongly chain local.

Theorem I. Suppose that $f$ is a function defined for p-solvable groups $G$, and assume that $f(G)=f(G / L)$ whenever $L$ is a normal $p^{\prime}$-subgroup of $G$. Then $f$ is strongly chain local for $p$-solvable groups.

For example, consider the following four isomorphism-constant functions:

(a) $f_{1}(G)=\left|G: \mathbf{O}^{p}(G)\right|$,

(b) $f_{2}(G)$ is the number of classes of $p$-elements in $G$,

(c) $f_{3}(G)$ is the number of irreducible characters in the principal $p$-block of $G$, and

(d) $f_{4}(G)$ is the $p$-length of $G$, where $G$ is $p$-solvable.

By Theorem \, each of these functions is strongly chain local for $p$-solvable groups.

If the underlying family is all finite groups, the function $f_{1}$ is defined, but examples show that it is not chain local. The function $f_{2}$ is defined and by Theorem $\mathrm{E}(\mathrm{a})$, it is chain local, but one can check that $\left(f_{2}\right)^{2}$ is not chain local, so $f_{2}$ is not strongly chain local. Dade's ordinary conjecture (for blocks) implies that $f_{3}$ is chain local, but a computation establishes that $\left(f_{3}\right)^{2}$ is not chain local, so $f_{3}$ is definitely not strongly chain local. Finally the function $f_{4}$ is not even defined for all finite groups.

We mention also that for $p$-solvable groups, Theorem Iguarantees that the function that counts the $p$-special characters of $G$ is strongly chain local. We shall also see that the function that counts the $p^{\prime}$-special characters is strongly chain local, but this lies much deeper, and it is not a consequence of Theorem [.

Finally, our methods allow us to give an elementary proof of the following remarkable result. This is essentially a result of $\mathrm{P}$. Webb, and it also appears as Corollary 9.20 of [14, with a more complicated proof.

Theorem J. If $G$ is a finite group, then the generalized character

$$
\theta=\sum_{C \in \mathcal{R}}(-1)^{\operatorname{len}(C)}\left(1_{G_{C}}\right)^{G}
$$

vanishes on the p-singular elements of $G$.

\section{TheOREM A}

Recall that given a group $G$ having order divisible by $p$, we defined the element $B(G)$ of the free-abelian group $\mathbf{A}$ by setting

$$
B(G)=\sum_{C \in \mathcal{R}}(-1)^{\operatorname{len}(C)}\left[G_{C}\right],
$$


where $\mathcal{R}$ is a set of representatives of the orbits of the conjugation action of $G$ on the set of chains in $G$, and $G_{C}$ is the stabilizer of the chain $C$. Recall also that by definition, $\mathbf{B}$ is the subgroup of $\mathbf{A}$ generated by all elements of the form $B(G)$.

The trivial chain (with length 0 ) must lie in $\mathcal{R}$, and its stabilizer is the full group $G$. It follows that one of the terms in the sum defining $B(G)$ is $[G]$, and hence we have

$$
[G]=B(G)-\sum_{C}(-1)^{\operatorname{len}(C)}\left[G_{C}\right]
$$

where here, the sum runs over the nontrivial chains $C$ in $\mathcal{R}$. Collecting terms corresponding to isomorphic chain stabilizers, we obtain an equation of the form $[G]=B(G)+\sum n_{i}\left[H_{i}\right]$, where the coefficients $n_{i}$ are nonzero integers, and the groups $H_{i}$ are pairwise nonisomorphic. Also, each of the groups $H_{i}$ is isomorphic to the stabilizer in $G$ of some nontrivial chain, and thus $\mathbf{O}_{p}\left(H_{i}\right)>1$. Since $B(G) \in \mathbf{B}$, we have $[G] \equiv \sum n_{i}\left[H_{i}\right] \bmod \mathbf{B}$, so to complete the proof of Theorem $\mathbf{A}$, it suffices to show that $\sum n_{i}=1$ and that the coefficients $n_{i}$ and the isomorphism types of the groups $H_{i}$ are uniquely determined. To do this, we will need two of the parts of Theorem E and we restate these here. (Proofs will be provided in later sections.) First, we recall Theorem $\mathrm{E}(\mathrm{h})$.

Lemma 2.1. The constant function $f(G)=1$ is chain local.

Now suppose that $[G] \equiv \sum n_{i}\left[H_{i}\right] \bmod \mathbf{B}$, as in Theorem $\mathbf{A}$ Since the function $f$ with constant value 1 is chain local by Lemma 2.1, we obtain equal results when we apply $f$ to the left and right sides of this congruence, and this yields $1=f(G)=$ $\sum n_{i} f\left(H_{i}\right)=\sum n_{i}$, as wanted.

Next, we restate TheoremE(e).

Theorem 2.2. Given a group $J$ with $\mathbf{O}_{p}(J)>1$, let $f_{J}(G)$ be the number of conjugacy classes of subgroups $X$ of $G$ such that $X \cong J$. Then the isomorphismconstant function $f_{J}$ is chain local.

In the following, we view the functions $f_{J}$ of Theorem 2.2 as homomorphisms from $\mathbf{A}$ to the integers $\mathbb{Z}$, and we write $\mathbf{J}$ to denote the intersection of the kernels of all of the homomorphisms $f_{J}$ for finite groups $J$ such that $\mathbf{O}_{p}(J)>1$. Theorem 2.2 asserts that $\mathbf{B} \subseteq \operatorname{ker}\left(f_{J}\right)$ whenever $\mathbf{O}_{p}(J)>1$, and it follows that $\mathbf{B} \subseteq \mathbf{J}$.

Theorem 2.3. We have $\mathbf{B}=\mathbf{J}$. Also, if $s \in \mathbf{B}$ is a linear combination of elements of the form $[J]$, where $\mathbf{O}_{p}(J)>1$, then $s=0$.

Proof. Suppose first that $s \in \mathbf{A}$ is a linear combination of the symbols $[J]$ for groups $J$ such that $\mathbf{O}_{p}(J)>1$. If $s \in \mathbf{J}$, we argue that $s=0$. To see this, suppose that $s \neq 0$, and write $s=\sum n_{i}\left[J_{i}\right]$ for $1 \leq i \leq r$, where the integer coefficients $n_{i}$ are all nonzero and the finite groups $J_{i}$ are pairwise nonisomorphic and satisfy the condition that $\mathbf{O}_{p}\left(J_{i}\right)>1$. By choice of notation, we can assume that $\left|J_{r}\right| \geq\left|J_{i}\right|$ for all $i$, and for notational simplicity, we write $J=J_{r}$.

Note that if $i<r$, then either $\left|J_{i}\right|<|J|$, or else $\left|J_{i}\right|=|J|$, but $J_{i}$ is not isomorphic to $J$. In either case, $J_{i}$ contains no isomorphic copy of $J$, and thus by the definition of $f_{J}$, we have $f_{J}\left(J_{i}\right)=0$. Also $f_{J}\left(J_{r}\right)=1$, and since we are assuming that $s \in \mathbf{J}$, we have $s \in \operatorname{ker}\left(f_{J}\right)$, and so

$$
0=f_{J}(s)=f_{J}\left(\sum n_{i}\left[J_{i}\right]\right)=n_{r}\left[J_{r}\right]
$$


It follows that $n_{r}=0$, and since this is a contradiction, we conclude that $s=0$, as claimed. The final assertion of the theorem now follows because $\mathbf{B} \subseteq \mathbf{J}$.

We have $\mathbf{B} \subseteq \mathbf{J}$, so to prove equality, we let $t \in \mathbf{J}$, and we work to show that $t \in \mathbf{B}$. Let $u$ be an element of the coset $\mathbf{B}+t \subseteq \mathbf{J}$. Write $u=\sum q_{j}\left[G_{j}\right]$, where the coefficients $q_{j}$ are integers, and let $a$ be the number of subscripts $j$ for which $\mathbf{O}_{p}\left(G_{j}\right)=1$. Finally, suppose that the coset representative $u$ is chosen so that $a$ is as small as possible.

If $a>0$, let $G$ be one of the groups $G_{j}$ such that $\mathbf{O}_{p}(G)=1$. We know that $[G]$ is congruent modulo $\mathbf{B}$ to a sum of the form $\sum n_{i}\left[H_{i}\right]$, where $\mathbf{O}_{p}\left(H_{i}\right)>1$. If we replace $[G]$ in the expression $\sum q_{j}\left[G_{j}\right]$ by $\sum n_{i}\left[H_{i}\right]$, we obtain another element $u^{\prime}$ of the coset $\mathbf{B}+t$, and $u^{\prime}$ is a linear combination of symbols $\left[G_{j}\right]$, where exactly $a-1$ of the groups $G_{j}$ fail to have a nontrivial normal $p$-subgroup. This contradicts the minimality of $a$, and we deduce that $a=0$, and thus $u$ is a linear combination of symbols $[J]$ such that $\mathbf{O}_{p}(J)>1$. Since $u \in \mathbf{J}$, the first part of the proof guarantees that $u=0$, and thus $\mathbf{B}+t=\mathbf{B}+u=\mathbf{B}$, and we have $t \in \mathbf{B}$, as required.

To complete the proof of Theorem $\mathrm{A}$, we must show that the coefficients $n_{i}$ and the symbols $\left[H_{i}\right]$ in the theorem are uniquely determined. To see this, suppose that $[G] \equiv \sum n_{i}\left[H_{i}\right] \bmod \mathbf{B}$, where the groups $H_{i}$ are pairwise nonisomorphic, and suppose also that $[G] \equiv \sum m_{i}\left[K_{i}\right] \bmod \mathbf{B}$, where the $K_{i}$ are pairwise nonisomorphic. Assume also that the groups $H_{i}$ and $K_{i}$ have nontrivial normal $p$-subgroups, and write

$$
s=\sum n_{i}\left[H_{i}\right]-\sum m_{i}\left[K_{i}\right]
$$

so $s \in \mathbf{B}$. By Theorem 2.3, we deduce that $s=0$, and so since $\mathbf{A}$ is free on the symbols $[X]$, we see that after appropriate renumbering, we have $n_{i}=m_{i}$ and $\left[H_{i}\right]=\left[K_{i}\right]$, as required. Once we establish Lemma 2.1 and Theorem 2.2, therefore, the proof of Theorem $\mathrm{A}$ will be complete.

\section{When ARE $[G]$ AND $[H]$ CONGRUent MOD B?}

In this section, we assume that $G$ and $H$ have orders divisible by $p$, and (assuming Theorem 2.2) we establish Theorems B and C, which address the question of when it is true that $[G] \equiv[H] \bmod \mathbf{B}$. The assertion of Theorem $\mathbf{B}$ is that this happens if and only if $f_{J}(G)=f_{J}(H)$ for all finite groups $J$ such that $\mathbf{O}_{p}(J)>1$. By the definition of $\mathbf{J}$, this latter condition is equivalent to saying that $[G] \equiv[H] \bmod \mathbf{J}$, and because Theorem 2.3 guarantees that $\mathbf{B}=\mathbf{J}$, we see that Theorem $\mathrm{B}$ follows without further proof.

In the case where $H \subseteq G$, an alternative necessary and sufficient condition to have $[G] \equiv[H] \bmod \mathbf{B}$ is provided by Theorem $\mathbf{C}$ which asserts that this congruence holds if and only if $\mathbf{N}_{G}(Q) \subseteq H$ for every nonidentity $p$-subgroup $Q$ of $H$.

We begin with a fairly standard preliminary result.

Lemma 3.1. Let $H \subseteq G$, where $p$ divides $|H|$. Then the following are equivalent:

(1) If $g \in G$ and $\left|H \cap H^{g}\right|$ is divisible by $p$, then $g \in H$.

(2) $\mathbf{N}_{G}(Q) \subseteq H$ for every nonidentity p-subgroup $Q$ of $H$.

Proof. First, assume (1), and suppose that $Q \subseteq H$ is a nonidentity $p$-subgroup. We must show that $\mathbf{N}_{G}(Q) \subseteq H$, so suppose $g \in \mathbf{N}_{G}(Q)$, and observe that $Q=Q^{g} \subseteq$ $H^{g}$, so $Q \subseteq H \cap H^{g}$. Then $p$ divides $\left|H \cap H^{g}\right|$, and thus $g \in H$, by (1). This proves that $\mathbf{N}_{G}(Q) \subseteq H$, as wanted. 
Conversely now, assume (2). Suppose that $p$ divides $\left|H \cap H^{g}\right|$ for some element $g \in G$, so we must show that $g \in H$. Write $D=H \cap H^{g}$, and let $S \in \operatorname{Syl}_{p}(D)$. Then $S$ is a nonidentity $p$-subgroup of $H$, and hence $\mathbf{N}_{G}(S) \subseteq H$ by (2). Also, $S \subseteq H^{g}$, so $S^{g^{-1}} \subseteq H$, and thus $\left(\mathbf{N}_{G}(S)\right)^{g^{-1}}=\mathbf{N}_{G}\left(S^{g^{-1}}\right) \subseteq H$ by $(2)$. Then $\mathbf{N}_{G}(S) \subseteq H^{g}$, and so $\mathbf{N}_{G}(S) \subseteq D$, and we deduce that $S$ is a full Sylow $p$-subgroup of $H$.

Now, $S^{g^{-1}} \subseteq H$ and $\left|S^{g^{-1}}\right|=|S|$, so $S^{g^{-1}}$ is also a Sylow $p$-subgroup of $H$, and therefore, $S^{g^{-1}}=S^{h}$ for some element $h \in H$. Then $h g \in \mathbf{N}_{G}(S) \subseteq H$, so $g \in H$, as required.

Proof of Theorem $\mathrm{C}$. First, assume that $\mathbf{N}_{G}(Q) \subseteq H$ for all nonidentity $p$-subgroups $Q$ of $H$. Fix $P \in \operatorname{Syl}_{p}(H)$, and observe that $P>1$ because $p$ divides $|H|$. By assumption, $\mathbf{N}_{G}(P) \subseteq H$, and it follows that $P$ is a full Sylow $p$-subgroup of $G$.

We must prove that $[G] \equiv[H] \bmod \mathbf{B}$, and we see by Theorem $\mathbf{B}$, that it suffices to show that $f_{J}(G)=f_{J}(H)$, for every finite group $J$ such that $\mathbf{O}_{p}(J)>1$. We fix $J$, and we argue that every subgroup $X$ of $G$ that is isomorphic to $J$ is conjugate in $G$ to some subgroup of $H$. To see this, observe that $\mathbf{O}_{p}(X)$ is conjugate to a subgroup of $P$, so replacing $X$ by a conjugate if necessary, we can assume that $\mathbf{O}_{p}(X) \subseteq P \subseteq H$. Now $\mathbf{O}_{p}(X) \cong \mathbf{O}_{p}(J)>1$, and thus we have $X \subseteq \mathbf{N}_{G}\left(\mathbf{O}_{p}(X)\right) \subseteq H$, where the final containment holds by assumption. We have now shown that as claimed, every subgroup of $G$ that is isomorphic to $J$ is $G$-conjugate to a subgroup of $H$.

By the result of the previous paragraph, we can choose a set $\mathcal{S}$ of representatives of the $G$-conjugacy classes of subgroups of $G$ that are isomorphic to $J$, where each member of $\mathcal{S}$ is a subgroup of $H$. We argue next that $\mathcal{S}$ is also a set of representatives of the $H$-conjugacy classes of subgroups of $H$ isomorphic to $J$. The members of $\mathcal{S}$ are contained in $H$, and since distinct members of $\mathcal{S}$ are not $G$-conjugate, they are certainly not $H$-conjugate. It suffices, therefore, to show that if $X$ is a subgroup of $H$ that is isomorphic to $J$, then $X$ is $H$-conjugate to some member of $\mathcal{S}$. By the choice of $\mathcal{S}$, we know that $X^{g}$ lies in $\mathcal{S}$, for some element $g \in G$, so it is enough to show that $X$ and $X^{g}$ are conjugate in $H$.

Now $X^{g} \subseteq H^{g}$ and since $X^{g} \in \mathcal{S}$, we also have $X^{g} \subseteq H$. Then $X^{g} \subseteq H \cap H^{g}$, and since $p$ divides $|J|=\left|X^{g}\right|$, it follows that $p$ divides $\left|H \cap H^{g}\right|$. By Lemma 3.1 we have $g \in H$, so $X$ and $X^{g}$ are conjugate in $H$, as wanted.

We now know that $\mathcal{S}$ is simultaneously a set of representatives of the classes of subgroups of $G$ that are isomorphic to $J$ and of the classes of subgroups of $H$ that are isomorphic to $J$, and thus $f_{J}(G)=|\mathcal{S}|=f_{J}(H)$, as required.

Conversely now, assume that $[G] \equiv[H] \bmod \mathbf{B}$, so by Theorem $\mathrm{B}$, we have $f_{J}(G)=f_{J}(H)$ for every finite group $J$ such that $\mathbf{O}_{p}(J)>1$. Taking $J$ to be a Sylow $p$-subgroup of $G$, we see that $H$ contains an isomorphic copy of $J$, and thus $H$ contains a Sylow $p$-subgroup $P$ of $G$.

Given a $p$-subgroup $U$ of $H$, we claim that every $G$-conjugate of $U$ that is contained in $H$ is $H$-conjugate to $U$. We can assume that $U>1$, and we let $\mathcal{A}$ be the set of $H$-classes of subgroups of $H$ that are isomorphic to $U$. Similarly, we let $\mathcal{B}$ be the set of $G$-classes of subgroups of $G$ that are isomorphic to $U$, and we observe that since $\mathbf{O}_{p}(U)=U>1$, we have $|\mathcal{A}|=f_{U}(H)=f_{U}(G)=|\mathcal{B}|$.

Each conjugacy class of subgroups of $H$ is contained in a unique conjugacy class of subgroups of $G$, so containment defines a map $\tau$ from $\mathcal{A}$ to $\mathcal{B}$, and we argue that $\tau$ is surjective. To see this, let $L$ be a member of $\mathcal{B}$, so $L$ is a conjugacy class 
of $p$-subgroups of $G$, and hence some member $T$ of $L$ is a subgroup of the Sylow subgroup $P$ of $G$. Now $P \subseteq H$, so $T \subseteq H$, and thus if $K$ is the class of subgroups of $H$ that contains $T$, we have $K \subseteq L$. Then $\tau(K)=L$, so $\tau$ is surjective, as wanted. We have seen that $|\mathcal{A}|=|\mathcal{B}|$, and it follows that $\tau$ is injective, and hence different $H$-classes of subgroups isomorphic to $U$ cannot be contained in the same $G$-class. It follows that as claimed, every $G$-conjugate of $U$ that is contained in $H$ is $H$-conjugate to $U$.

Let $Q$ be a nonidentity $p$-subgroup of $H$, and let $N=\mathbf{N}_{G}(Q)$. We must show that $N \subseteq H$, so we assume that this is false, and we work to obtain a contradiction. Choose $Q$ so that $N \nsubseteq H$, and subject to this condition, assume that $|N|$ is as large as possible.

Now suppose that $M \subseteq H$ and $M \cong N$, and let $U=\mathbf{O}_{p}(M)$, so $U \cong \mathbf{O}_{p}(N) \supseteq Q$, and thus $U>1$. We argue now that $\mathbf{N}_{G}(U) \subseteq H$. This is clear if $\mathbf{N}_{G}(U)=M$, so we can suppose that $\left|\mathbf{N}_{G}(U)\right|>|M|=|N|$, and thus by the maximality of $N$, we have $\mathbf{N}_{G}(U) \subseteq H$, as wanted.

Suppose now that $M^{g} \subseteq H$ for some element $g \in G$. Then $U^{g} \subseteq H$, and as we have seen, it follows that $U^{g}=U^{h}$ for some element $h \in H$. We thus have $g h^{-1} \in \mathbf{N}_{G}(U) \subseteq H$, and so $g \in H$.

Let $\mathcal{S}$ be a set of representatives of the $H$-conjugacy classes of subgroups $M$ of $H$ such that $M \cong N$. No two distinct members of $\mathcal{S}$ are conjugate in $H$, and it follows by the foregoing argument that no two distinct members of $\mathcal{S}$ can be conjugate in $G$. Now $|\mathcal{S}|=f_{N}(H)=f_{N}(G) \geq|\mathcal{S}|$, where the second equality holds because $\mathbf{O}_{p}(N)>1$. Equality thus holds, and thus $N$ must be $G$-conjugate to some member of $\mathcal{S}$. We can thus write $N^{g} \subseteq H$ for some element $g \in G$, and we have $Q^{g} \subseteq H$. By an earlier argument, $Q^{g}=Q^{h}$ for some element $h \in H$, so we have $N^{h}=\mathbf{N}_{G}\left(Q^{h}\right)=\mathbf{N}_{G}\left(Q^{g}\right)=N^{g} \subseteq H$, and it follows that $N \subseteq H$. This is the desired contradiction.

We have now proved Theorems $\mathrm{A}$, B, and C, assuming Lemma 2.1, and Theorem 2.2

\section{Computations with chains}

In this section, we prove that certain isomorphism-constant functions are chain local. Recall that by our definition, an isomorphism-constant function $f$ with values in an abelian group $U$ is chain local if when we view $f$ as a homomorphism from the free-abelian group $\mathbf{A}$ into $U$, we have $\mathbf{B} \subseteq \operatorname{ker}(f)$.

For the purposes of this section, it is convenient to set aside temporarily the free-abelian group A. Instead, we use the equivalent condition that $f$ is chain local precisely when the chain sum for $f$ vanishes, or in other words,

$$
\sum_{C \in \mathcal{R}}(-1)^{\operatorname{len}(C)} f\left(G_{C}\right)=0
$$

for every finite group $G$ (in the underlying family) having order divisible by $p$, where $\mathcal{R}$ is a set of representatives of the orbits of the conjugation action of $G$ on its chains.

This point of view gives us the freedom to consider functions $f$ defined only on the set of subgroups of order divisible by $p$ of a given group $G$. Also, it allows us to relax the condition that $f$ is isomorphism constant, and to assume only that $f$ is conjugation constant on $G$, which means that for subgroups $X$ and $Y$ of $G$ 
having orders divisible by $p$, we have $f(X)=f(Y)$ whenever $X$ and $Y$ are conjugate in $G$. (Observe that this condition is sufficient to guarantee that the chain sum for $f$ on $G$ is independent of the choice of the representative set $\mathcal{R}$.) We say that the conjugation-constant function $f$ on $G$ is chain local on $G$ if the chain sum for $f$ on $G$ vanishes.

Given an isomorphism-constant function $f$ and a group $G$ having order divisible by $p$, we see that the restriction $\alpha$ of $f$ to the set of subgroups of $G$ having order divisible by $p$ is a conjugation-constant function on $G$. (We will usually use Latin letters for isomorphism-constant functions and Greek letters for conjugationconstant functions defined on subgroups of some fixed group $G$.) Continuing to assume that $f$ is isomorphism constant, we observe that $f$ is chain local if and only if the restrictions of $f$ to $G$ are chain local on $G$ for all groups $G$ having order divisible by $p$.

Next, we present several preliminary results that will enable us to evaluate certain chain sums. The first of these is a fairly standard tool for working with chains.

Lemma 4.1. Suppose $C$ is a chain in $G$, and let $Q$ be a nontrivial p-subgroup of G. Assume that either

(1) $Q$ normalizes every member of $C$ or

(2) every member of $C$ normalizes $Q$.

Then $C$ and $Q$ determine a chain $C^{*}$ such that the condition corresponding to (1) or (2) is satisfied with $C^{*}$ in place of $C$. Also, either $C$ is a proper subset of $C^{*}$ or $C^{*}$ is a proper subset of $C$, and the following hold:

(a) $\operatorname{len}\left(C^{*}\right)=\operatorname{len}(C) \pm 1$.

(b) $\left(C^{*}\right)^{*}=C$.

Furthermore, every subgroup of $G$ stabilizing $C$ and normalizing $Q$ also stabilizes $C^{*}$. In particular, if $Q \triangleleft G$, the stabilizers of $C$ and $C^{*}$ in $G$ are equal.

Note that our notation is somewhat deficient because it does not indicate that the chain $C^{*}$ depends on the subgroup $Q$ as well as on the chain $C$. We trust, however, that this will not create confusion.

Proof of Lemma 4.1. Write $C=\left\{P_{i} \mid 0 \leq i \leq n\right\}$, where $n=\operatorname{len}(C)$ and

$$
1=P_{0}<P_{1}<\cdots<P_{n} .
$$

Since $Q \nsubseteq P_{0}$, there exists a unique maximum subscript $m$, with $0 \leq m \leq \operatorname{len}(C)$, and such that $Q \nsubseteq P_{m}$. In particular, we have $P_{m}<P_{m} Q$, and also $P_{m} Q \subseteq P_{i}$ whenever $m<i \leq n$.

If the $p$-subgroup $P_{m} Q$ is not a member of the chain $C$, then since $P_{m}<P_{m} Q$ and $P_{m} Q<P_{i}$ for all subscripts $i$ such that $m<i \leq n$, we can insert the $p$-group $P_{m} Q$ into the chain $C$, and we define $C^{*}=C \cup\left\{P_{m} Q\right\}$. (Note that if $m=\operatorname{len}(C)$, it would be more accurate to say that $C^{*}$ is obtained from $C$ by "appending", rather than by "inserting" the group $P_{m} Q$.) Observe that in this case, $C^{*}$ is a chain of length $n+1$, and $C^{*}$ properly contains $C$. (We write $C^{*} \subset C$.) On the other hand, if $P_{m} Q$ is a member of $C$, we can delete it, and we define $C^{*}=C-\left\{P_{m} Q\right\}$, so in this case, $C^{*}$ is a chain of length $n-1$, and $C^{*}$ is properly contained in $C$.

Note that if each member of $C$ normalizes $Q$, or if $Q$ normalizes each member of $C$, the same condition holds with $C^{*}$ in place of $C$. We can thus use the same procedure to construct the chain $\left(C^{*}\right)^{*}$. In either case, where $C \subset C^{*}$ or $C^{*} \subset C$, we see that $C$ and $C^{*}$ agree on all terms $P_{i}$ with $0 \leq i \leq m$, so none of these terms 
in the chain $C^{*}$ contains $Q$. The following term in $C^{*}$ (if there is one) does contain $Q$, and thus $P_{m}$ is the largest term in $C^{*}$ that fails to contain $Q$. It follows that if $P_{m} Q$ is in $C^{*}$, then $\left(C^{*}\right)^{*}=C^{*}-\left\{P_{m} Q\right\}=C$, and if $P_{m} Q$ is not in $C^{*}$, then $\left(C^{*}\right)^{*}=C^{*} \cup\left\{P_{m} Q\right\}=C$, so in all cases $\left(C^{*}\right)^{*}=C$.

Corollary 4.2. Suppose that $\mathbf{O}_{p}(G)>1$. Then every conjugacy-constant function on $G$ is chain local.

Proof. Let $Q=\mathbf{O}_{p}(G)>1$, so condition (2) of Lemma 4.1 applies, and we have a map $C \mapsto C^{*}$ on chains. This map defines a bijection from the set of even-length chains on $G$ to the set of odd-length chains on $G$, and each of these sets of chains is invariant under conjugation by elements of $G$. Furthermore, because $Q \triangleleft G$, it follows that $\left(C^{g}\right)^{*}=\left(C^{*}\right)^{g}$, and in particular, the chain stabilizers $G_{C}$ and $G_{C^{*}}$ are equal.

Now choose a set $\mathcal{R}$ of representatives of the $G$-orbits of even-length chains, and note that $\mathcal{R}^{*}=\left\{C^{*} \mid C \in \mathcal{R}\right\}$ is a set of representatives of the $G$-orbits of odd-length chains. Given a conjugacy-constant function $\alpha$ on $G$, therefore, the corresponding chain sum is

$$
\sum_{C \in \mathcal{R}}(-1)^{\operatorname{len}(C)} \alpha\left(G_{C}\right)+\sum_{C \in \mathcal{R}}(-1)^{\operatorname{len}\left(C^{*}\right)} \alpha\left(G_{C^{*}}\right)
$$

and this sum vanishes because $\operatorname{len}\left(C^{*}\right)=\operatorname{len}(C) \pm 1$ and $G_{C}=G_{C^{*}}$. We conclude that $\alpha$ is chain local on $G$, as required.

We are now ready to introduce our principal tool for evaluating chain sums. Given a group $G$, let $C$ be a chain in $G$ and let $P$ be a nontrivial $p$-subgroup of $G$. Let $X$ be a subset of $\mathbf{N}_{G}(P)$, and assume that $P$ and $X$ are contained in the stabilizer $G_{C}$ of $C$. In this situation, we say that $(C, P, X)$ is a normalizing triple.

Note that $G$ acts by conjugation on its set of normalizing triples. For each orbit $\mathcal{O}$ of the action of $G$ on this set, the left components of the members of $\mathcal{O}$ form an orbit of the action of $G$ on its chains, so the lengths of all of the chains appearing as left components of members of $\mathcal{O}$ are equal, and we write $s(\mathcal{O})=(-1)^{\operatorname{len}(C)}$, where $C$ is any chain that occurs as a left component of a member of $\mathcal{O}$. Also, if $\mathcal{R}$ is a set of representatives of the $G$-orbits of chains, we see that for each $G$-orbit $\mathcal{O}$ of normalizing triples, exactly one member of $\mathcal{R}$ appears as a left component of a member of $\mathcal{O}$, and thus the orbit $\mathcal{O}$ determines a unique member of $\mathcal{R}$.

Now let $(C, P, X)$ be a normalizing triple. Since $P$ stabilizes $C$, it follows by Lemma 4.1 that $C$ and $P$ uniquely determine a chain $C^{*}$. Also, since $X$ normalizes both $C$ and $P$, we see that $X$ also normalizes $C^{*}$, and thus $\left(C^{*}, P, X\right)$ is a normalizing triple that is uniquely determined by the triple $(C, P, X)$, Writing $(C, P, X)^{*}=\left(C^{*}, P, X\right)$, we see that $*$ defines a permutation on the set of normalizing triples.

If $\mathcal{O}$ is a $G$-orbit of normalizing triples, we write

$$
\mathcal{O}^{*}=\left\{(C, P, X)^{*} \mid(C, P, X) \in \mathcal{O}\right\} \text {. }
$$

We see that $\mathcal{O}^{*}$ is an orbit of normalizing triples because $\left((C, P, X)^{g}\right)^{*}=\left((C, P, X)^{*}\right)^{g}$ for all elements $g \in G$. Also $\left|\mathcal{O}^{*}\right|=|\mathcal{O}|$, and since $s\left(\mathcal{O}^{*}\right)=-s(\mathcal{O})$, we see that $\mathcal{O}^{*} \neq \mathcal{O}$. We observe that $\left(\mathcal{O}^{*}\right)^{*}=\mathcal{O}$, and we refer to $\mathcal{O}$ and $\mathcal{O}^{*}$ as paired orbits.

Lemma 4.3. Let $(C, P, X)$ be a normalizing triple. Write $(C, P, X)^{*}=\left(C^{*}, P, X\right)$, and let $H=G_{C}$ and $H^{*}=G_{C^{*}}$. The following then hold: 
(a) If $X=\mathbf{N}_{H}(P)$, then $X=\mathbf{N}_{H^{*}}(P)$.

(b) If $X=\mathbf{N}_{H}(P)$ and $P \in \operatorname{Syl}_{p}(H)$, then $P \in \operatorname{Syl}_{p}\left(H^{*}\right)$.

(c) If $P \in \operatorname{Syl}_{p}\left(\mathbf{C}_{H}(X)\right)$, then $P \in \operatorname{Syl}_{p}\left(\mathbf{C}_{H^{*}}(X)\right)$.

Proof. Since $(C, P, X)$ and $\left(C^{*}, P, X\right)$ are normalizing triples, each of $P$ and $X$ is contained in both $H$ and $H^{*}$. We have $\mathbf{N}_{H}(P)=X \subseteq H^{*}$, so $\mathbf{N}_{H}(P) \subseteq \mathbf{N}_{H^{*}}(P)$. We must prove that equality holds here, so it suffices to show that $\mathbf{N}_{H^{*}}(P) \subseteq H$. Now $\mathbf{N}_{H^{*}}(P)$ stabilizes both $P$ and $C^{*}$, so it stabilizes $\left(C^{*}\right)^{*}=C$, and thus $\mathbf{N}_{H^{*}}(P) \subseteq H$, proving (a).

For (b), we assume that $P \in \operatorname{Syl}_{p}(H)$. If $C \subset C^{*}$, then $P \subseteq H^{*} \subseteq H$, and it is clear that $P \in \operatorname{Syl}_{p}\left(H^{*}\right)$, as required.

We can suppose, therefore, that $C^{*} \subset C$, so $P \subseteq H \subseteq H^{*}$, and since $P \in$ $\operatorname{Syl}_{p}(H)$, it suffices to show that $\mathbf{N}_{H^{*}}(P) \subseteq H$. By hypothesis, $\mathbf{N}_{H}(P)=X$, so by (a), we have $\mathbf{N}_{H^{*}}(P)=X \subseteq H$, as required.

Finally, for (c), write $U=\mathbf{C}_{H}(X)$ and $U^{*}=\mathbf{C}_{H^{*}}(X)$. If $C \subset C^{*}$, then $H^{*} \subseteq H$, so $U^{*}=U \cap H^{*} \supseteq P$. Then $P \subseteq U^{*} \subseteq U$, and since we are assuming that $P \in \operatorname{Syl}_{p}(U)$, it is clear that $P \in \operatorname{Syl}_{p}\left(U^{*}\right)$, as required.

We can suppose, therefore, that $C^{*} \subset C$, so $H \subseteq H^{*}$, and thus $P \subseteq U \subseteq U^{*}$. Since $P \in \operatorname{Syl}_{p}(U)$, we see that to show that $P \in \operatorname{Syl}_{p}\left(U^{*}\right)$, it suffices to show that $\mathbf{N}_{U^{*}}(P) \subseteq U$. Now $\mathbf{N}_{U^{*}}(P) \subseteq U^{*} \subseteq H^{*}$, so $\mathbf{N}_{U^{*}}(P)$ stabilizes both $C^{*}$ and $P$, and thus $\mathbf{N}_{U^{*}}(P)$ stabilizes $\left(C^{*}\right)^{*}=C$. Then $\mathbf{N}_{U^{*}}(P) \subseteq H$, and we have $\mathbf{N}_{U^{*}}(P) \subseteq H \cap U^{*}=U$, as wanted.

The following is somewhat technical, but it is completely elementary. Lemma 4.4(a) and (b) are essential for most of the main results in this section, but Lemma 4.4(c) will not be used until we prove Corollary 4.10, which is Theorem J]

Lemma 4.4. Let $G$ be a finite group, and let $\mathcal{T}$ be a $G$-invariant set of normalizing triples in $G$. Given a chain $C$ with stabilizer $H$ in $G$, let $\mathcal{Q}$ be the (possibly empty) set of pairs $(Q, Y)$ such that $(C, Q, Y)$ lies in $\mathcal{T}$, and note that $\mathcal{Q}$ is $H$-invariant.

(a) Let $\mathcal{U}$ be an $H$-orbit on $\mathcal{Q}$. Then there is a unique $G$-orbit $\mathcal{O}$ on $\mathcal{T}$ such that the set

$$
\mathcal{S}=\{(C, Q, Y) \mid(Q, Y) \in \mathcal{U}\}
$$

is contained in $\mathcal{O}$, and we write $\tau(\mathcal{U})=\mathcal{O}$.

(b) The map $\tau$ in (a) is a bijection from the set of $H$-orbits on $\mathcal{Q}$ onto the set of those $G$-orbits $\mathcal{O}$ on $\mathcal{T}$ such that $C$ is the left component of some member of $\mathcal{O}$.

(c) Let $\mathcal{U}$ be an $H$-orbit in $\mathcal{Q}$ and let $\mathcal{O}=\tau(\mathcal{U})$ as in (b). Then $|\mathcal{O}|=\mid G$ : $H|| \mathcal{U} \mid$.

Proof. Observe first that $\mathcal{S} \subseteq \mathcal{T}$. Also, by hypothesis, $\mathcal{U}$ is an orbit on $\mathcal{Q}$, so $\mathcal{U}$ is nonempty, and thus $\mathcal{S}$ is nonempty. Now $H$ stabilizes $C$ and acts transitively on $\mathcal{U}$, and thus $H$ acts transitively on $\mathcal{S}$. It follows that $\mathcal{S}$ is contained in a unique $G$-orbit $\mathcal{O}$ on $\mathcal{T}$, and this defines the map $\tau$. Also, since $\mathcal{S} \subseteq \mathcal{O}$, we see that $\mathcal{O}$ contains a triple with left component $C$.

To show that $\tau$ is injective, suppose $\mathcal{V}$ is an $H$-orbit on $\mathcal{Q}$ such that $\tau(\mathcal{U})=\mathcal{O}=$ $\tau(\mathcal{V})$. Let $(P, X) \in \mathcal{U}$ and $(Q, Y) \in \mathcal{V}$, so by the definition of $\tau$, both $(C, P, X)$ and $(C, Q, Y)$ lie in the $G$-orbit $\mathcal{O}$, and hence there exists an element $g \in G$ such that $(C, P, X)^{g}=(C, Q, Y)$. Then $g$ stabilizes $C$, so $g \in H$, and also $(P, X)^{g}=(Q, Y)$, 
so $(P, X)$ and $(Q, Y)$ lie in the same $H$-orbit on $\mathcal{Q}$. It follows that $\mathcal{U}=\mathcal{V}$, and thus $\tau$ is injective, as required.

To see that $\tau$ maps onto the set of $G$-orbits on $\mathcal{T}$ that contain a triple with left component $C$, let $\mathcal{O}$ be such an orbit, and suppose $(C, Q, Y) \in \mathcal{O}$. Since $(C, Q, Y) \in \mathcal{T}$, we have $(Q, Y) \in \mathcal{Q}$, and thus $(Q, Y)$ lies in some $H$-orbit $\mathcal{U}$ on $\mathcal{Q}$. Then $\tau(\mathcal{U})=\mathcal{O}$, and the proof of (b) is complete.

For (c), suppose $\tau(\mathcal{U})=\mathcal{O}$, and let $(Q, Y) \in \mathcal{U}$. Let $N$ be the stabilizer in $H$ of $(Q, Y)$, so $|\mathcal{U}|=|H| /|N|$. Now $(C, Q, Y) \in \mathcal{O}$, and $N$ is the stabilizer in $G$ of the triple $(C, Q, Y)$, and thus

$$
|\mathcal{O}|=\frac{|G|}{|N|}=\frac{|G|}{|H|} \frac{|H|}{|N|}=|G: H||\mathcal{U}|,
$$

as required.

Next, we use Lemma 4.4 to construct some chain-local conjugacy-constant functions.

Theorem 4.5. Fix a nontrivial p-subgroup $P$ of a group $G$ and a subset $X$ of $G$. Let $\alpha_{(P, X)}, \beta_{(P, X)}, \gamma_{(P, X)}, \delta_{(P, X)}$, and $\epsilon_{(P, X)}$ be the integer-valued conjugacyconstant functions on $G$ defined as follows for subgroups $H$ of $G$ such that $|H|$ is divisible by $p$ :

(a) $\alpha_{(P, X)}(H)$ is the number of $H$-orbits of pairs $(Q, Y)$ that are $G$-conjugate to $(P, X)$, where $Q \subseteq H$ and $Y \subseteq \mathbf{N}_{H}(Q)$.

(b) $\beta_{(P, X)}(H)$ is the number of $H$-orbits of pairs $(Q, Y)$ that are $G$-conjugate to $(P, X)$, where $Q \subseteq H$ and $Y=\mathbf{N}_{H}(Q)$.

(c) $\gamma_{(P, X)}(H)$ is the number of $H$-orbits of pairs $(Q, Y)$ that are $G$-conjugate to $(P, X)$, where $Q \subseteq H$ and $Y=\mathbf{N}_{H}(Q)$, and also $Q=\mathbf{O}_{p}(Y)$.

(d) $\delta_{(P, X)}(H)$ is the number of $H$-orbits of pairs $(Q, Y)$ that are $G$-conjugate to $(P, X)$, where $Q \subseteq H$ and $Y=\mathbf{N}_{H}(Q)$, and also $Q$ is a Sylow p-subgroup of $H$.

(e) $\epsilon_{(P, X)}(H)$ is the number of $H$-orbits of pairs $(Q, Y)$ that are $G$-conjugate to $(P, X)$, where $Y \subseteq H$ and $Q$ is a Sylow p-subgroup of $\mathbf{C}_{H}(Y)$.

Then $\alpha_{(P, X)}, \beta_{(P, X)}, \gamma_{(P, X)}, \delta_{(P, X)}$, and $\epsilon_{(P, X)}$ are chain-local functions on $G$.

Proof. Note that in all five function definitions, $Y$ normalizes $Q$, and both $Y$ and $Q$ are contained in the subgroup $H$. If $H$ is the stabilizer in $G$ of some chain $C$, therefore, we see that $(C, Q, Y)$ is a normalizing triple. We propose to apply Lemma 4.4(a),(b), so we define five $G$-invariant sets of normalizing triples that will play the role of $\mathcal{T}$ in the lemma.

(1) $\mathcal{T}_{1}$ is the set of normalizing triples $(C, Q, Y)$ such that $(Q, Y)$ is $G$-conjugate to $(P, X)$.

(2) $\mathcal{T}_{2}$ is the set of normalizing triples $(C, Q, Y)$ such that $(Q, Y)$ is $G$-conjugate to $(P, X)$, and also $Y=\mathbf{N}_{G_{C}}(Q)$.

(3) $\mathcal{T}_{3}$ is the set of normalizing triples $(C, Q, Y)$ such that $(Q, Y)$ is $G$-conjugate to $(P, X)$ and also $Y=\mathbf{N}_{G_{C}}(Q)$ and $Q=\mathbf{O}_{p}(Y)$.

(4) $\mathcal{T}_{4}$ is the set of normalizing triples $(C, Q, Y)$ such that $(Q, Y)$ is $G$-conjugate to $(P, X)$, and also $Y=\mathbf{N}_{G_{C}}(Q)$ and $Q \in \operatorname{Syl}_{p}\left(G_{C}\right)$.

(5) $\mathcal{T}_{5}$ is the set of normalizing triples $(C, Q, Y)$ such that $(Q, Y)$ is $G$-conjugate to $(P, X)$, and also $Q \in \operatorname{Syl}_{p}\left(\mathbf{C}_{G_{C}}(Y)\right)$. 
If $(C, Q, Y)$ lies in $\mathcal{T}_{1}$, then since $(C, Q, Y)$ is a normalizing triple, it follows that $(C, Q, Y)^{*}$ is defined, and it too lies in $\mathcal{T}_{1}$. Theorem 4.3(a) guarantees that if $(C, Q, Y)$ lies in $\mathcal{T}_{2}$, then $(C, Q, Y)^{*}$ lies in $\mathcal{T}_{2}$ and similarly for $\mathcal{T}_{3}$. Also, if $(C, Q, Y)$ lies in $\mathcal{T}_{4}$, then $(C, Q, Y)^{*}$ lies in $\mathcal{T}_{4}$ by Theorem $4.3(\mathrm{~b})$, and by Theorem 4.3(c), we see that if $(C, Q, Y)$ lies in $\mathcal{T}_{5}$, then $(C, Q, Y)^{*}$ also lies in $\mathcal{T}_{5}$. Thus for each $G$-orbit $\mathcal{O}$ on any of the sets $\mathcal{T}_{i}$, the paired orbit $\mathcal{O}^{*}$ is also contained in $\mathcal{T}_{i}$.

Simplifying the notation, we drop the subscript $(P, X)$, and we write $\alpha, \beta, \gamma$, $\delta$, and $\epsilon$ for the five conjugacy-constant functions we have defined. Also, we temporarily fix the chain $C$.

By the definition of the set $\mathcal{T}_{1}$, we see that $\alpha\left(G_{C}\right)$ is the number of $G_{C}$-orbits of pairs $(Q, Y)$ such that $(C, Q, Y)$ lies in $\mathcal{T}_{1}$. Also, $\beta\left(G_{C}\right)$ is the number of $G_{C}$-orbits of pairs $(Q, Y)$ such that the triple $(C, Q, Y)$ lies in $\mathcal{T}_{2}$, and similarly for $\gamma, \delta$, and $\epsilon$.

It follows by Lemma 4.4(a) and (b) that

(1) $\alpha\left(G_{C}\right)$ is the number of $G$-orbits $\mathcal{O}$ in $\mathcal{T}_{1}$, such that $\mathcal{O}$ contains a triple with left component $C$.

(2) $\beta\left(G_{C}\right)$ is the number of $G$-orbits $\mathcal{O}$ in $\mathcal{T}_{2}$, such that $\mathcal{O}$ contains a triple with left component $C$.

(3) $\gamma\left(G_{C}\right)$ is the number of $G$-orbits $\mathcal{O}$ in $\mathcal{T}_{3}$, such that $\mathcal{O}$ contains a triple with left component $C$.

(4) $\delta\left(G_{C}\right)$ is the number of $G$-orbits $\mathcal{O}$ in $\mathcal{T}_{4}$, such that $\mathcal{O}$ contains a triple with left component $C$.

(5) $\epsilon\left(G_{C}\right)$ is the number of $G$-orbits $\mathcal{O}$ in $\mathcal{T}_{5}$, such that $\mathcal{O}$ contains a triple with left component $C$.

Recall now that if $C$ is the left component of a member of a $G$-orbit $\mathcal{O}$ of normalizing triples, then $(-1)^{\operatorname{len}(C)}=s(\mathcal{O})$. It follows that

$$
(-1)^{\operatorname{len}(C)} \alpha\left(G_{C}\right)=\sum_{\mathcal{O}} s(\mathcal{O})
$$

where the sum runs over all of those $G$-orbits $\mathcal{O}$ in $\mathcal{T}_{1}$ that contain a member having left component equal to $C$. Similar formulas hold for $\beta, \gamma, \delta$, and $\epsilon$, where we sum over the $G$-orbits in $\mathcal{T}_{2}, \mathcal{T}_{3}, \mathcal{T}_{4}$, and $\mathcal{T}_{5}$, respectively, that contain a member with left component $C$.

Now let $\mathcal{R}$ be a set of representatives of the $G$-orbits of chains. For every $G$-orbit $\mathcal{O}$ on $\mathcal{T}_{1}$, there is exactly one chain $C$ in $\mathcal{R}$ such that $C$ is the left component of a member of $\mathcal{O}$. Summing the above over all members of $\mathcal{R}$, therefore, we see that each $G$-orbit in $\mathcal{T}_{1}$ is counted exactly once, and so we have

$$
\sum_{C \in \mathcal{R}}(-1)^{\operatorname{len}(C)} \alpha\left(G_{C}\right)=\sum_{\mathcal{O}} s(\mathcal{O})
$$

where the sum on the right runs over all $G$-orbits $\mathcal{O}$ in $\mathcal{T}_{1}$. Similarly, the chain sums corresponding to the functions $\beta, \gamma, \delta$, and $\epsilon$ are equal to sums of the form $\sum s(\mathcal{O})$, where $\mathcal{O}$ runs over the $G$-orbits in $\mathcal{T}_{2}, \mathcal{T}_{3}, \mathcal{T}_{4}$, and $\mathcal{T}_{5}$, respectively.

Every $G$-orbit $\mathcal{O}$ of triples in $\mathcal{T}_{i}$ for $i \in\{1,2,3,4,5\}$ is paired with a unique $G$ orbit $\mathcal{O}^{*}$, and as we have seen, this paired orbit also lies in $\mathcal{T}_{i}$. Since $s(\mathcal{O})=-s\left(\mathcal{O}^{*}\right)$, it follows that the chain sums corresponding to the functions $\alpha, \beta, \gamma, \delta$, and $\epsilon$ vanish, and hence each of these conjugacy constant functions is chain local on $G$, as required. 
Corollary 4.6. Let $N$ be a finite group, and let $f_{N}, g_{N}, r_{N}$, and $s_{N}$ be the isomorphism-constant functions defined below for groups $G$ having order divisible by $p$. Then $f_{N}$ is chain local if $\mathbf{O}_{p}(N)>1$, and the functions $g_{N}, r_{N}$, and $s_{N}$ are chain local unconditionally.

(a) $f_{N}(G)$ is the number of conjugacy classes of subgroups $X$ of $G$ such that $X \cong N$.

(b) $g_{N}(G)$ is the number of conjugacy classes of nontrivial p-subgroups $Q$ of $G$ such that $\mathbf{N}_{G}(Q) \cong N$.

(c) $r_{N}(G)$ is the number of conjugacy classes of nontrivial radical p-subgroups $Q$ of $G$ such that $\mathbf{N}_{G}(Q) \cong N$.

(d) $s_{N}(G)=1$ if $\mathbf{N}_{G}(P) \cong N$, where $P \in \operatorname{Syl}_{p}(G)$, and $s_{N}(G)=0$, otherwise.

Note that Corollary 4.6(a) is exactly TheoremE(e), which was restated as Theorem 2.2. Also, Corollary 4.6(b) is Theorem E(f), and Corollary 4.6(c) is Theorem $\mathrm{E}(\mathrm{g})$. Finally, we remark that if $\mathbf{O}_{p}(N)=1$, then the functions $g_{N}, r_{N}$, and $s_{N}$ are identically 0 , and hence they are trivially chain local. (This is obvious for $g_{N}$ and $r_{N}$, and since by assumption $p$ divides $|G|$, we see that if $\mathbf{O}_{p}(N)=1$, then $N$ cannot be isomorphic to the normalizer of a Sylow $p$-subgroup of $G$, so $s_{N}(G)=0$.)

Proof of Corollary 4.6. Fix a group $G$ having order divisible by $p$, and let $\alpha, \beta, \gamma$, and $\delta$ be the restrictions of $f_{N}, g_{N}, r_{N}$, and $s_{N}$, respectively, to the subgroups of $G$. It suffices to show for every choice of $G$ that each of these four conjugacy-constant functions is chain local on $G$.

Let $\mathcal{S}$ be a set of representatives of the $G$-orbits of subgroup pairs $(Q, Y)$ of $G$, where $Q$ is a nonidentity $p$-subgroup, $Q \triangleleft Y$ and $Y \cong N$. Also, recall that for (a), the hypothesis guarantees that $\mathbf{O}_{p}(N)>1$, and as we have seen, for (b), (c), and (d), we can assume without loss that $\mathbf{O}_{p}(N)>1$.

Let $H$ be a subgroup of $G$ of order divisible by $p$. Each $H$-orbit $K$ of subgroups $Y \subseteq H$ such that $Y \cong N$ uniquely determines a set of pairs

$$
\mathcal{A}_{K}=\left\{(Q, Y) \mid Y \in K \text { and } Q=\mathbf{O}_{p}(Y)\right\},
$$

and we see that $H$ acts transitively on $\mathcal{A}_{K}$.

Now let $\mathcal{P}_{1}$ be the set of pairs $(Q, Y)$ such that

(1) $Q=\mathbf{O}_{p}(Y)$,

(2) $Y \cong N$,

(3) $Y \subseteq H$,

and observe that the map $K \mapsto \mathcal{A}_{K}$ is a bijection from the set of $H$-orbits of subgroups $Y \subseteq H$ such that $Y \cong N$ onto the set of $H$-orbits on $\mathcal{P}_{1}$. Also note that if $(Q, Y)$ is an arbitrary member of $\mathcal{P}_{1}$, then $Q=\mathbf{O}_{p}(Y) \cong \mathbf{O}_{p}(N)>1$.

By definition, $\alpha(H)$ is the number of $H$-orbits of subgroups $Y$ of $H$ such that $Y \cong N$, and so we see that $\alpha(H)$ is the number of $H$-orbits on $\mathcal{P}_{1}$. Also, every member of each $H$-orbit on $\mathcal{P}_{1}$ is $G$-conjugate to some unique member $(P, X)$ of $\mathcal{S}$, where $(P, X)$ has the property that $P=\mathbf{O}_{p}(X)$. The number of $H$-orbits on $\mathcal{P}_{1}$ whose members are $G$-conjugate to some given member $(P, X)$ of $\mathcal{S}$ is $\alpha_{(P, X)}(H)$, where $\alpha_{(P, X)}$ is the function of Theorem 4.5(a), and it follows that

$$
\alpha(H)=\sum_{(P, X)} \alpha_{(P, X)}(H),
$$

where the sum runs over members $(P, X) \in \mathcal{S}$ such that $P=\mathbf{O}_{p}(X)$. 
Since $(P, X) \in \mathcal{S}$, we have $P>1$, and it follows by Theorem 4.5 that the function $\alpha_{(P, X)}$ is chain local on $G$. We conclude that $\alpha$ is chain local, and this completes the proof of (a).

For (b), recall that by definition, $\beta(H)$ is the number of $H$-orbits of nontrivial $p$-subgroups $Q$ of $H$ such that $\mathbf{N}_{H}(Q) \cong N$. Each such $H$-orbit $K$ uniquely determines a set of pairs

$$
\mathcal{A}_{K}=\left\{(Q, Y) \mid Q \in K \text { and } Y=\mathbf{N}_{H}(Q)\right\}
$$

and we see that $H$ acts transitively on $\mathcal{A}_{K}$.

Now let $\mathcal{P}_{2}$ be the set of pairs $(Q, Y)$ such that

(1) $Y=\mathbf{N}_{H}(Q)$,

(2) $Q$ is a nonidentity $p$-subgroup of $H$,

(3) $Y \cong N$,

and observe that the map $K \mapsto \mathcal{A}_{K}$ is a bijection from the set of $H$-orbits of subgroups $Q \subseteq H$ such that $\mathbf{N}_{H}(Q) \cong N$ onto the set of $H$-orbits on $\mathcal{P}_{2}$.

Then $\beta(H)$ is the number of $H$-orbits on the set $\mathcal{P}_{2}$. Also, every member of each $H$-orbit on $\mathcal{P}_{2}$ is $G$-conjugate to some unique member $(P, X)$ of $\mathcal{S}$. The number of $H$-orbits on $\mathcal{P}_{2}$ whose members are $G$-conjugate to some given member $(P, X)$ of $\mathcal{S}$ is $\beta_{(P, X)}(H)$, where $\beta_{(P, X)}$ is the function of Theorem 4.5(b), and it follows that

$$
\beta(H)=\sum_{(P, X) \in \mathcal{S}} \beta_{(P, X)}(H) .
$$

Theorem 4.5 guarantees that for each member $(P, X)$ of $\mathcal{S}$, the function $\beta_{(P, X)}$ is chain local on $G$, so $\beta$ is a sum of chain local functions, and thus $\beta$ is chain local, as required, proving (b).

Recall next that $\gamma(H)$ is the number of $H$-orbits of nontrivial $p$-subgroups $Q$ of $H$ such that $\mathbf{N}_{H}(Q) \cong N$, with the additional requirement that $Q$ is radical in $H$, or equivalently $Q=\mathbf{O}_{p}\left(\mathbf{N}_{H}(Q)\right)$. As in the proof of (b), each such $H$-orbit $K$ uniquely determines a set of pairs

$$
\mathcal{A}_{K}=\left\{(Q, Y) \mid Q \in K \text { and } Y=\mathbf{N}_{H}(Q)\right\},
$$

where $H$ acts transitively on $K$.

Now let $\mathcal{P}_{3}$ be the set of pairs $(Q, Y)$ such that

(1) $Y=\mathbf{N}_{H}(Q)$,

(2) $Q=\mathbf{O}_{p}(Y)$

(3) $Y \cong N$,

and observe that the map $K \mapsto \mathcal{A}_{K}$ is a bijection from the set of $H$-orbits of subgroups $Q \subseteq H$ such that $\mathbf{N}_{H}(Q) \cong N$ and $Q=\mathbf{O}_{p}\left(\mathbf{N}_{H}(Q)\right)$, onto the set of $H$-orbits on $\mathcal{P}_{3}$.

Then $\gamma(H)$ is the number of $H$-orbits on the set $\mathcal{P}_{3}$. Also, every member of each $H$-orbit on $\mathcal{P}_{3}$ is $G$-conjugate to some unique member $(P, X)$ of $\mathcal{S}$. The number of $H$-orbits on $\mathcal{P}_{3}$ whose members are $G$-conjugate to some given member of $(P, X)$ of $\mathcal{S}$ is $\gamma_{(P, X)}(H)$, where $\gamma_{(P, X)}$ is the function of Theorem 4.5(c), and it follows that

$$
\gamma(H)=\sum_{(P, X) \in \mathcal{S}} \gamma_{(P, X)}(H)
$$


Theorem 4.5 guarantees that for each member $(P, X)$ of $\mathcal{S}$, the function $\gamma_{(P, X)}$ is chain local on $G$, so $\gamma$ is a sum of chain local functions, and thus $\gamma$ is chain local, as required, proving (c).

By definition, $\delta(H)=1$ if $\mathbf{N}_{H}(Q) \cong N$, where $Q \in \operatorname{Syl}_{p}(H)$, and $\delta(H)=0$, otherwise. By Sylow's theorem, therefore, $\delta(H)$ is the number of $H$-orbits on the set $\mathcal{P}_{4}$ of pairs $(Q, Y)$ such that

(1) $Y=\mathbf{N}_{H}(Q)$,

(2) $Q \in \operatorname{Syl}_{p}(H)$

(3) $Y \cong N$.

Reasoning as before, it follows that $\delta$ is the sum of functions of the form $\delta_{(P, X)}$, as in Theorem $4.5(\mathrm{~d})$, where $(P, X)$ runs over the set $\mathcal{S}$. By Theorem 4.5 , the functions $\delta_{(P, X)}$ are chain local on $G$, and hence $\delta$ is chain local.

The proofs of Theorems E(e), E(f), and E(g) are now complete, and the following is a restatement of Theorem $\mathrm{E}(\mathrm{i})$.

Corollary 4.7. Let $f_{d}(G)$ denote the number of conjugacy classes of $G$ that have defect $d$, where $d>0$. Then the isomorphism-constant function $f_{d}$ is chain local.

Proof. Fix a group $G$ having order divisible by $p$, and let $\alpha_{d}$ be the conjugacyconstant function on $G$ obtained by restricting $f_{d}$ to the set of subgroups of $G$ having order divisible by $p$. It suffices to show that $\alpha_{d}$ is chain local on $G$.

Let $H \subseteq G$, where $|H|$ is divisible by $p$. Each conjugacy class $K$ of elements of $H$ uniquely determines a set of pairs

$$
\mathcal{A}_{K}=\left\{(Q, y) \mid y \in K \text { and } Q \in \operatorname{Syl}_{p}\left(\mathbf{C}_{H}(y)\right)\right\},
$$

and we see that $H$ acts transitively by conjugation on $\mathcal{A}_{K}$. Also, if the class $K$ has defect $d$, then for each pair $(Q, y)$ in $\mathcal{A}_{K}$, we have $|Q|=p^{d}$.

Now let $\mathcal{P}$ be the set of pairs $(Q, y)$ such that

(1) $y \in H$,

(2) $Q \in \operatorname{Syl}_{p}\left(\mathbf{C}_{H}(y)\right)$,

and observe that the map $K \mapsto \mathcal{A}_{K}$ is a natural bijection from the set of conjugacy classes of $H$ onto the set of $H$-orbits on $\mathcal{P}$, and thus $\alpha_{d}(H)$ is the number of $H$-orbits on $\mathcal{P}$ that correspond to classes having defect $d$.

Now let $\mathcal{S}$ be a set of representatives of the $G$-orbits of pairs $(P, x)$, where $x \in G$ and $P \subseteq G$ is a nonidentity $p$-subgroup that centralizes $x$. All members of each $H$-orbit on $\mathcal{P}$ are $G$-conjugate to some unique member $(P, x)$ of $\mathcal{S}$, and we see that the number of $H$-orbits on $\mathcal{P}$ whose members are $G$-conjugate to a given pair $(P, x)$ in $\mathcal{S}$ is equal to $\epsilon_{(P, x)}(H)$, where $\epsilon_{(P, x)}$ is the function $\epsilon_{(P, X)}$ of Theorem 4.5(e) and $X=\{x\}$. It follows that

$$
\alpha_{d}(H)=\sum_{(P, x)} \epsilon_{(P, x)}(H),
$$

where the sum runs over those members $(P, x)$ of $\mathcal{S}$ such that $|P|=p^{d}$. We are assuming that $d>0$, so $P$ is a nonidentity $p$-group, and hence Theorem 4.5(e) guarantees that the functions $\epsilon_{(P, x)}$ are chain local on $G$. It follows that $\alpha_{d}$ is chain local, as required.

Our next result includes Theorem $\mathrm{E}(\mathrm{b})$ and most of Theorem $\mathrm{E}(\mathrm{a})$. 
Corollary 4.8. Fix a set $T$ of integers divisible by $p$, and let $f$ be the isomorphismconstant function that counts the conjugacy classes of elements of $G$ having orders in the set $T$. Then $f$ is chain local.

If $T$ is the set of all multiples of $p$, then $f$ counts the $p$-singular classes of $G$, and the fact that $f$ is chain local is Theorem $\mathrm{E}(\mathrm{b})$. If we take $T$ to be the set of all powers of $p$ exceeding 1 , then $f$ counts the classes of nonidentity $p$-elements of $G$, so the total number of classes of elements of $G$ that have $p$-power order is $f(G)+1$. In order to deduce Theorem $\mathrm{E}$ (a) from Corollary 4.8, therefore, we would need to establish that the constant function $f(G)=1$ is chain local. This fact was stated as Theorem $\mathrm{E}(\mathrm{h})$, and again as Lemma 2.1, but we have not yet presented a proof.

Proof of Corollary 4.8. Suppose $G$ is a group having order divisible by $p$, and let $\mu$ be the conjugacy-constant function on $G$ obtained by restricting $f$ to subgroups of $G$. It suffices, therefore, to show that $\mu$ is chain local on $G$ for every choice of $G$.

Let $H \subseteq G$, where $p$ divides $|H|$, and suppose that $K$ is a conjugacy class of $H$ consisting of elements having order divisible by $p$. Then $K$ uniquely determines the set of pairs

$$
\mathcal{A}_{K}=\left\{(Q, y) \mid y \in K \text { and } Q=\mathbf{O}_{p}(\langle y\rangle)\right\},
$$

so $H$ acts transitively by conjugation on $\mathcal{A}_{K}$, and we observe that if $(Q, y) \in \mathcal{A}_{K}$, then $Q>1$ since $\langle y\rangle$ is cyclic and by assumption, the order of $y$ is divisible by $p$.

Now let $\mathcal{P}$ be the set of pairs $(Q, y)$ such that

(1) $y \in H$,

(2) $Q=\mathbf{O}_{p}(\langle y\rangle)>1$,

and observe that the map $K \mapsto \mathcal{A}_{K}$ is a bijection from the set of classes of $H$ consisting of elements having order divisible by $p$ onto the set of $H$-orbits on $\mathcal{P}$.

Now let $\mathcal{S}$ be a set of representatives of the $G$-orbits of pairs $(P, x)$, where $P$ is a nonidentity $p$-subgroup of $G$ and $x \in \mathbf{N}_{G}(P)$. Then every member of each $H$-orbit on $\mathcal{P}$ is $G$-conjugate to some unique member of $\mathcal{S}$, and we see that the number of $H$-orbits on $\mathcal{P}$ whose members are $G$-conjugate to a given pair $(P, x)$ in $\mathcal{S}$ is equal to $\alpha_{(P, x)}(H)$, where $\alpha_{(P, x)}$ is the chain-local function $\alpha_{(P, X)}$ of Theorem 4.5(a) and $X=\{x\}$.

Now $f(H)$ is the number of classes of elements of $H$ that have orders in the set $T$, and it follows that

$$
f(H)=\sum_{(P, x)} \alpha_{(P, x)}(H),
$$

where the sum runs over those pairs $(P, x) \in \mathcal{S}$ such that the order of $x$ lies in $T$. Since the functions $\alpha_{(P, x)}$ are chain local, it follows that $f$ is chain local, as required.

As our final application of the techniques of this section, we prove the following, which will yield Theorem J.

Theorem 4.9. Fix an element $x$ having order divisible by $p$ in a group $G$, and let $\alpha_{x}$ be the integer-valued conjugacy-constant function for $G$ defined by letting $\alpha_{x}(H)$ be the number of right cosets $H t$ of $H$ in $G$ such that Htx $=H t$. Then $\alpha_{x}$ is chain local on $G$.

Proof. Let $X=\{x\}$ and $P=\mathbf{O}_{p}(\langle x\rangle)$, and note that $P>1$ because we are assuming that $x$ has order divisible by $p$. Also, let $\mathcal{T}$ be the set of normalizing 
triples $(C, Q, Y)$ in $G$ such that $(Q, Y)$ is $G$-conjugate to $(P, X)$, and note that $\mathcal{T}$ is $G$-invariant, so we can apply Lemma 4.4. Observe also, that for every $G$-orbit $\mathcal{O}$ on $\mathcal{T}$, the paired orbit $\mathcal{O}^{*}$ is also a $G$-orbit on $\mathcal{T}$.

Now fixing a chain $C$, we write $H=G_{C}$, and we let $\mathcal{Q}$ be the (possibly empty) $H$ invariant set of pairs $(Q, Y)$ such that $(C, Q, Y)$ lies in $\mathcal{T}$. For each pair $(Q, Y) \in \mathcal{Q}$, we have $Y=\{y\}$, where $y \in H$ and $y$ is a member of the set $S$ of elements of $H$ that are conjugate to $x$ in $G$. In fact, $S$ is exactly the set of elements $y$ of $H$ such that $\{y\}$ is the right component of some pair in $\mathcal{Q}$. To see this, observe that if $y \in S$, and $Q=\mathbf{O}_{p}(\langle y\rangle)$, then $(Q,\{y\})$ is the unique pair in $\mathcal{Q}$ with right component $\{y\}$.

Now $S$ is the union of some set $\mathcal{K}$ of classes of $H$, and each member $K$ of $\mathcal{K}$ uniquely determines a set of pairs

$$
\mathcal{U}_{K}=\left\{(Q, Y) \mid Y=\{y\}, \text { where } y \in K \text { and } Q=\mathbf{O}_{p}(\langle y\rangle)\right\},
$$

where $\mathcal{U}_{K} \subseteq \mathcal{Q}$, and $|K|=\left|\mathcal{U}_{K}\right|$. Also, since $K$ is a conjugacy class of $H$, we see that $H$ acts transitively on $\mathcal{U}_{K}$, and thus the map $K \mapsto \mathcal{U}_{K}$ is a bijection from $\mathcal{K}$ onto the set of $H$-orbits on $\mathcal{Q}$.

By Lemma 4.4(a), there is a bijection $\tau$ from the set of $H$-orbits on $\mathcal{Q}$ onto the set of those $G$-orbits $\mathcal{O}$ on $\mathcal{T}$ such that $C$ is the left component of some member of $\mathcal{O}$. Given $K \in \mathcal{K}$, we write $\tau\left(\mathcal{U}_{K}\right)=\mathcal{O}_{K}$, and we observe that Lemma 4.4(b) yields

$$
\left|\mathcal{O}_{K}\right|=|G: H|\left|\mathcal{U}_{K}\right|=|G: H||K|
$$

Now consider the chain sum

$$
a=\sum_{C \in \mathcal{R}}(-1)^{\operatorname{len}(c))} \alpha_{x}\left(G_{C}\right)
$$

where $\mathcal{R}$ is a set of representatives of the $G$-orbits of chains. To show that $\alpha_{x}$ is chain local on $G$, we must show that $a=0$, so we proceed to compute the term in the sum that corresponds to the given chain $C$. For notational convenience, we write $\alpha=\alpha_{x}$, and we recall that we have defined $H=G_{C}$. We begin by computing $\alpha(H)$.

We see that $\alpha(H)=m /|H|$, where $m$ is the number of elements $t \in G$ such that $H t x=H t$, or equivalently, $t x t^{-1} \in H$, and thus $t x t^{-1} \in S$. For each element $y \in S$, the number of elements $t \in G$ such that $t x t^{-1}=y$ is exactly $\left|\mathbf{C}_{G}(x)\right|$, so $m=\left|\mathbf{C}_{G}(x)\right||S|$, and we have

$$
\begin{aligned}
\alpha(H)=\frac{m}{|H|}=\frac{\left|\mathbf{C}_{G}(x)\right||S|}{|H|}=\frac{\left|\mathbf{C}_{G}(x)\right|}{|G|}|G: H||S| & =\frac{\left|\mathbf{C}_{G}(x)\right|}{|G|}|G: H| \sum_{K \in \mathcal{K}}|K| \\
& =\frac{\left|\mathbf{C}_{G}(x)\right|}{|G|} \sum_{K \in \mathcal{K}}\left|\mathcal{O}_{K}\right| .
\end{aligned}
$$

Recall now that the map $K \mapsto \mathcal{O}_{K}$ is a bijection from the set $\mathcal{K}$ onto the set of all $G$-orbits on $\mathcal{T}$ that contain a triple with left component $C$. The final sum above, therefore, is equal to $\sum|\mathcal{O}|$, where $\mathcal{O}$ runs over all $G$-orbits on $\mathcal{T}$ that contain a triple with left component $C$. We thus have

$$
\alpha\left(G_{C}\right)=\frac{\left|\mathbf{C}_{G}(x)\right|}{|G|} \sum_{\mathcal{O}}|\mathcal{O}| .
$$


For each $G$-orbit $\mathcal{O}$ on $\mathcal{T}$, there is a unique member of the representative set $\mathcal{R}$ that occurs as a left component of a member of $\mathcal{O}$, and we see now that

$$
a=\sum_{C \in \mathcal{R}}(-1)^{\operatorname{len}(C)} \alpha\left(G_{C}\right)=\frac{\left|\mathbf{C}_{G}(x)\right|}{|G|} \sum_{\mathcal{O}} s(\mathcal{O})|\mathcal{O}|,
$$

where here, the sum on the right runs over all $G$-orbits on $\mathcal{T}$. Then $a=0$, as required, because for each orbit $\mathcal{O}$ on $\mathcal{T}$, the paired orbit $\mathcal{O}^{*}$ is also one of the terms in the sum, and we have $\left|\mathcal{O}^{*}\right|=|\mathcal{O}|$ and $s\left(\mathcal{O}^{*}\right)=-s(\mathcal{O})$.

Next, we restate and prove Theorem J], which is essentially a result of Webb.

Corollary 4.10. If $G$ is a finite group, then the generalized character

$$
\theta=\sum_{C \in \mathcal{R}}(-1)^{\operatorname{len}(C)}\left(1_{G_{C}}\right)^{G}
$$

vanishes on the p-singular elements of $G$.

Proof. Observe that $\left(1_{G_{C}}\right)^{G}$ is the permutation character corresponding to the right multiplication action of $G$ on the set of right cosets of $G_{C}$ in $G$. Given a $p$-singular element $x \in G$, therefore, we see that $\theta(x)$ is exactly the chain sum for the conjugacy-constant function $\alpha_{x}$ defined in Theorem 4.9. Since $\alpha_{x}$ is chain local, this chain sum vanishes.

In 18 , Webb defined what he called the "Steinberg module" of a group $G$, and this corresponds to the negative of the generalized character $\theta$ in Corollary 4.10 . Webb's "module" is virtually projective, and this implies that the corresponding generalized character vanishes at $p$-singular elements.

\section{Functions For Which $f(G)=f(N)$}

We return now to the point of view of the introduction, where isomorphismconstant functions defined on the underlying family $\mathcal{F}$, and having values in an abelian group $U$, can be viewed as homomorphisms from the free-abelian group $\mathbf{A}$ into $U$. Also, we recall that the isomorphism-constant functions that are chain local for $\mathcal{F}$ correspond exactly to those homomorphisms that vanish on the subgroup $\mathbf{B}$ of $\mathbf{A}$.

The following powerful result is Theorem $\mathrm{F}$ (a).

Theorem 5.1. Let $f$ be an isomorphism-constant function with values in some abelian group $U$. For every finite group $G$ that lies in the underlying set $\mathcal{F}$ and has order divisible by $p$, assume that $f(G)=f(N)$, where $N$ is the normalizer of a Sylow p-subgroup of $G$. Then $f$ is chain local for $\mathcal{F}$.

Proof. Given a finite group $M$, let $s_{M}$ be the integer-valued isomorphism-constant function of Corollary 4.6(d), so $s_{M}$ is chain local, and by definition, $s_{M}(G)=1$ if $M$ is isomorphic to the normalizer of a Sylow $p$-subgroup of $G$, and $s_{M}(G)=0$, otherwise.

Now let $G$ lie in $\mathcal{F}$ and have order divisible by $p$. Let $N$ be the normalizer of a Sylow $p$-subgroup of $G$, and note that $N \in \mathcal{F}$, so $f(N)$ is defined. We argue now that

$$
f(G)=f(N)=\sum_{[M]} s_{M}(G) f(M)
$$


where the sum runs over all isomorphism classes $[M]$ of groups $M$ lying in $\mathcal{F}$. The first equality holds by hypothesis, and the second equality is clear since $s_{M}(G)=0$ unless $M \cong N$, in which case $s_{M}(G)=1$.

Now let $b \in \mathbf{B}$, so we can write $b=\sum n_{i}\left[X_{i}\right]$, where the sum is finite, the coefficients $n_{i}$ lie in $\mathbb{Z}$, and the groups $X_{i}$ lie in $\mathcal{F}$. Then

$$
\begin{aligned}
f(b)=\sum_{i} n_{i} f\left(X_{i}\right)=\sum_{i} n_{i} \sum_{[M]} s_{M}\left(X_{i}\right) f(M) & =\sum_{[M]} \sum_{i} n_{i} s_{M}\left(X_{i}\right) f(M) \\
& =\sum_{[M]} s_{M}(b) f(M) \\
& =0,
\end{aligned}
$$

where the final equality holds because the functions $s_{M}$ are chain local, and thus $s_{M}(b)=0$ for all $M$. Then $f(b)=0$ for all $b \in \mathbf{B}$, and we conclude that $f$ is chain local for $\mathcal{F}$, as required.

We now present some consequences of Theorem 5.1. The first of these is the following, which is Theorem $\mathrm{E}(\mathrm{h})$, and which was restated as Lemma 2.1. Also, recall that this is the final ingredient needed to complete the proof of Theorem $\mathrm{A}$ and to derive Theorem $\mathrm{E}$ (a) from Corollary 4.8

Corollary 5.2. The constant function $f(G)=1$ for groups $G$ having order divisible by $p$ is chain local.

Proof. If $N$ is the normalizer of a Sylow $p$-subgroup of $G$, we have $f(G)=1=f(N)$, so $f$ is chain local by Theorem 5.1.

Next, we recall that an isomorphism constant function $f$ with values in an abelian group $U$ is "strongly chain local" if all of the functions $f_{(u)}$ are chain local for elements $u \in U$, where by definition $f_{(u)}(G)=1$ if $f(G)=u$ and $f_{(u)}(G)=0$, otherwise. (Also, if we wish to mention the underlying family $\mathcal{F}$ explicitly, we say that $f$ is strongly chain local for $\mathcal{F}$ if each of the functions $f_{(u)}$ is chain local for $\mathcal{F}$.)

We saw that strongly chain local functions are guaranteed to be chain local, but that not every chain local function is strongly chain local. We have the following, however.

Corollary 5.3. Let $f$ be as in Theorem 5.1, so $f$ has values in an abelian group $U$, and $f(G)=f(N)$ whenever $N$ is the normalizer of a Sylow p-subgroup of $G$, where $G$ lies in the underlying set $\mathcal{F}$ and $G$ has order divisible by $p$. Then $f$ is strongly chain local for $\mathcal{F}$.

Proof. Let $u \in U$, and let $N$ be the normalizer of a Sylow $p$-subgroup of $G$, where $G$ lies in $\mathcal{F}$ and $|G|$ is divisible by $p$. By hypothesis, there exists an element $v \in U$ such that $f(G)=v=f(N)$, so if $u=v$, we have $f_{(u)}(G)=1=f_{(u)}(N)$, and otherwise, $f_{(u)}(G)=0=f_{(u)}(N)$. In both cases, therefore, $f_{(u)}(G)=f_{(u)}(N)$, and hence Theorem 5.1 guarantees that $f_{(u)}$ is chain local for $\mathcal{F}$. It follows by definition that $f$ is strongly chain local for $\mathcal{F}$.

Now let $m$ be the McKay function, so $m(G)$ is the number of irreducible characters of $G$ having degrees not divisible by $p$. Recall that the McKay conjecture posits that $m(G)=m(N)$ for all groups $G$, where $N$ is the normalizer of a Sylow $p$-subgroup of $G$. If the McKay conjecture is true, therefore, then Theorem 5.1 
applies, and we deduce that the function $m$ is chain local, and in fact, if the McKay conjecture is true, then $m$ is strongly chain local by Corollary 5.3. This proves Theorem $\mathrm{H}$.

Conversely, if $m$ is chain local, then the McKay conjecture holds. The following is Theorem $\mathrm{D}$.

Theorem 5.4. The McKay function $m$ is chain local if and only if $m(G)=m(N)$ for all finite groups $G$, where $N$ is the normalizer of a Sylow p-subgroup of $G$.

To prove this, we will use the fact that if $G$ is a minimal counterexample to the McKay conjecture, then $\mathbf{O}_{p}(G)=1$. This is proved by fairly standard charactertheoretic techniques in [14, where it appears as the first part of the proof of Theorem 9.27. (We mention that Theorem 9.27 of [14] asserts that Dade's ordinary conjecture implies the McKay conjecture. We will present a different proof of this fact following the proof of Theorem 5.4, below.) We need the following preliminary result.

Lemma 5.5. Suppose that $G$ is a finite group such that $p$ divides $|G|$. Also, let $f$ and $g$ be chain-local functions, and assume that $f(X)=g(X)$ for every subgroup $X$ of $G$ such that $\mathbf{O}_{p}(X)>1$. Then $f(G)=g(G)$.

Proof. By Theorem $\mathrm{A}$, there exist subgroups $H_{i}$ of $G$ such that $[G] \equiv \sum n_{i}\left[H_{i}\right]$ $\bmod \mathbf{B}$, where $\mathbf{O}_{p}\left(H_{i}\right)>1$. By assumption, $f\left(H_{i}\right)=g\left(H_{i}\right)$ for all $i$, and since $f$ and $g$ are chain local, we have

$$
f(G)=\sum n_{i} f\left(H_{i}\right)=\sum n_{i} g\left(H_{i}\right)=g(G)
$$

as required.

Proof of Theorem 5.4. As we have seen, Theorem 5.1 guarantees that if the McKay conjecture is true, then the function $m$ is chain local. It suffices, therefore, to assume that $m$ is chain local, and to prove that $m(G)=m(N)$ for all finite groups $G$, where $N$ is the normalizer of a Sylow $p$-subgroup of $G$. Assuming that this is false, let $G$ be a minimal counterexample, and note that $N \neq G$ because $m(N) \neq m(G)$, and thus $|G|$ is divisible by $p$. Also, as we remarked, the minimality of $G$ guarantees that $\mathbf{O}_{p}(G)=1$.

Let $m_{0}$ be the isomorphism-constant function defined by setting $m_{0}(X)=m(M)$, where $M$ is the normalizer of a Sylow $p$-subgroup of $X$. Then $m_{0}(M)=m(M)=$ $m_{0}(X)$, and so $m_{0}$ is chain local by Theorem 5.1 .

Now if $X \subseteq G$ and $\mathbf{O}_{p}(X)>1$, then $X<G$ because $\mathbf{O}_{p}(G)=1$. By the minimality of $G$, therefore, we have $m(X)=m(M)=m_{0}(X)$, where as before, $M$ is the normalizer of a Sylow $p$-subgroup of $X$. By Lemma 5.5, therefore, $m(G)=$ $m_{0}(G)=m(N)$, and this is a contradiction.

Recall that by definition, the defect of a character $\chi \in \operatorname{Irr}(G)$ is the nonnegative integer $d$ such that $p^{d}$ is the $p$-part of $|G| / \chi(1)$. Also, $k_{d}(G)$ is the number of irreducible characters of $G$ that have defect $d$, and Dade's ordinary conjecture (in its block-free form) asserts that the functions $k_{d}$ are chain local for $d>0$.

Theorem 5.6. If the block-free form of Dade's ordinary conjecture is true, then the McKay conjecture is also true.

Proof. We must show that if $N$ is the normalizer of a Sylow $p$-subgroup of a group $G$, then $m(G)=m(N)$, so we assume that this is false, and we let $G$ be a minimal 
counterexample. Then $m(G) \neq m(N)$, and (as before) we conclude that $p$ divides $|G|$ and that $\mathbf{O}_{p}(G)=1$.

Let $p^{a}$ be the $p$-part of $|G|$, so $a>0$. If $X$ is a finite group and the $p$-part of $|X|$ is less than $p^{a}$, then $k_{a}(X)=0$. Also, if the $p$-part of $|X|$ is equal to $p^{a}$, then $k_{a}(X)=m(X)$.

Now let $f$ be the isomorphism-constant function defined by setting $f(X)=$ $k_{a}(Y)$, where $X$ is arbitrary and $Y$ is the normalizer of a Sylow $p$-subgroup of $X$. We see by Theorem 5.1 that $f$ is chain local.

Now let $H$ be a proper subgroup of $G$, and let $M$ be the normalizer of a Sylow $p$-subgroup of $H$, so $m(H)=m(M)$ by the minimality of $G$, and we argue that $f(H)=k_{a}(H)$. To see this, let $p^{b}$ be the $p$-part of $|H|$, so $b \leq a$ and $p^{b}$ is the $p$-part of $|M|$. Also, by definition, $f(H)=k_{a}(M)$.

Now if $b=a$, then

$$
f(H)=k_{a}(M)=m(M)=m(H)=k_{a}(H),
$$

as required, where the second and fourth equalities hold because the functions $m$ and $k_{a}$ agree on groups whose order has $p$-part equal to $p^{a}$. If $b<a$, on the other hand, we have

$$
f(H)=k_{a}(M)=0=k_{a}(H),
$$

so in all cases, $f(H)=k_{a}(H)$, as claimed.

Now $f$ is chain local, and since we are assuming Dade's conjecture, $k_{a}$ is also chain local. We have seen that $f$ and $k_{a}$ agree on proper subgroups of $G$, and since $\mathbf{O}_{p}(G)=1$, we deduce that $f$ and $k_{a}$ agree on all subgroups $H \subseteq G$ such that $\mathbf{O}_{p}(H)>1$. Lemma 5.5 thus guarantees that $k_{a}(G)=f(G)$. Then

$$
m(G)=k_{a}(G)=f(G)=k_{a}(N)=m(N),
$$

where the third equality holds by the definition of $f$. This is a contradiction.

\section{Functions that ARE DEFined LOCALly}

The following is Theorem $\mathrm{F}(\mathrm{b})$.

Theorem 6.1. Let $h$ be an arbitrary isomorphism-constant function with values in an abelian group $U$, and let

$$
f(G)=\sum_{Q} h\left(\mathbf{N}_{G}(Q)\right)
$$

where $Q$ runs over a set of representatives of the $G$-classes of nonidentity $p$-subgroups of $G$. Then $f$ is a chain-local function.

The function $f$ in Theorem 6.1 is defined in terms of the values of $h$ on what are traditionally referred to as the "local" subgroups of $G$, and so we can think of $f$ as being defined locally. The assertion of the theorem, therefore, is that an isomorphism-constant function that can be defined locally must be chain local. We shall see later that in fact, every chain local function can be defined locally.

Proof of Theorem 6.1. Let $g_{N}$ be the function defined in Corollary 4.6(b), so $g_{N}(G)$ is the number of conjugacy classes of nonidentity $p$-subgroups $Q$ of $G$ such that 
$\mathbf{N}_{G}(Q) \cong N$. Then $g_{N}(G)$ is the number of terms in the sum defining $f$ for which $\mathbf{N}_{G}(Q) \cong N$, and this yields

$$
f(G)=\sum_{[N]} g_{N}(G) h(N),
$$

where $[N]$ runs over the isomorphism classes of groups that occur as normalizers of nonidentity $p$-subgroups in $G$. Since $g_{N}(G)=0$ for groups $N$ that are not isomorphic to any of the subgroups of the form $\mathbf{N}_{G}(Q)$, it follows that in the above sum, we can allow $[N]$ to run over all isomorphism classes of groups.

Now let $b \in \mathbf{B}$, and write $b=\sum n_{i}\left[X_{i}\right]$, where the sum is finite and the coefficients $n_{i}$ are integers. Then

$$
\begin{aligned}
f(b)=\sum_{i} n_{i} f\left(X_{i}\right)=\sum_{i} n_{i} \sum_{[N]} g_{N}\left(X_{i}\right) h(N) & =\sum_{[N]} \sum_{i} n_{i} g_{N}\left(X_{i}\right) h(N) \\
& =\sum_{[N]} g_{N}(b) h(N) \\
& =0
\end{aligned}
$$

where the final equality holds because $g_{N}$ is chain local by Corollary 4.6, and thus $g_{N}(b)=0$. Then $f(b)=0$ for all $b \in \mathbf{B}$, and thus $f$ is chain local.

What is essentially the same argument proves the following, which is Theorem F(c). In this modification of Theorem 6.1, we sum over radical subgroups only, and this proves to be useful for some applications.

Theorem 6.2. Let $h$ be an arbitrary isomorphism-constant function with values in an abelian group $U$, and let

$$
f(G)=\sum_{Q} h\left(\mathbf{N}_{G}(Q)\right),
$$

where $Q$ runs over a set of representatives of the $G$-classes of nonidentity radical p-subgroups of $G$. Then the function $f$ is chain local.

Proof. The proof of Theorem 6.1 goes through with essentially no change except that in place of the function $g_{N}$ of Corollary 4.6(b), we use the function $r_{N}$ of Corollary 4.6(c). Recall that $r_{N}(G)$ is the number of $G$-classes of nonidentity radical $p$-subgroups $Q$ of $G$ such that $\mathbf{N}_{G}(Q) \cong N$.

As an application of Theorem 6.1, we produce a chain-local function that detects whether or not a group $G$ is $p$-nilpotent. Observe that the following does not really provide a new $p$-nilpotence criterion; it is simply a restatement of Frobenius' theorem in the language of chain-local functions.

Corollary 6.3. Let $f(G)$ be the number of conjugacy classes of p-subgroups $Q$ of $G$ such that $\mathbf{N}_{G}(Q)$ fails to be p-nilpotent. Then $f$ is chain local, and $G$ is p-nilpotent if and only if $f(G)=0$.

Proof. By the Frobenius normal p-complement theorem (Theorem 5.26 of [8]), $G$ is $p$-nilpotent if and only if $\mathbf{N}_{G}(Q)$ is $p$-nilpotent for every nonidentity $p$-subgroup $Q$ of $G$. It follows that $G$ is $p$-nilpotent if and only $f(G)=0$. 
Now let $h$ be the isomorphism-constant function defined by setting $h(X)=0$ if $X$ is $p$-nilpotent and $h(X)=1$, otherwise. Clearly then,

$$
f(G)=\sum_{Q} h\left(\mathbf{N}_{G}(Q)\right),
$$

where the sum runs over a set of representatives of the conjugacy classes of nonidentity $p$-subgroups $Q$ of $G$. It follows by Theorem 6.1 that $f$ is chain local, as required.

Corollary 6.4. Let $G$ have order divisible by $p$ and suppose $[G] \equiv \sum n_{i}\left[H_{i}\right] \bmod \mathbf{B}$, as in Theorem $\mathrm{A}$. Then $G$ is p-nilpotent if and only if each of the groups $H_{i}$ is p-nilpotent.

Proof. We know that the groups $H_{i}$ are isomorphic to subgroups of $G$, so if $G$ is $p$-nilpotent, all of the groups $H_{i}$ are also $p$-nilpotent. Conversely, assume that all of the groups $H_{i}$ are $p$-nilpotent, and let $f$ be the function of Corollary 7.3, so $f\left(H_{i}\right)=0$ for all $i$. Since $f$ is chain local, we have $f(G)=\sum n_{i} f\left(H_{i}\right)=0$, and thus $G$ is $p$-nilpotent by another application of Corollary 7.3 ,

We have now established all of Theorem E except for $E(c)$ and $E(d)$, which involve Brauer blocks. Before we present proofs of these two results, we offer a very brief review of a few relevant facts from block theory.

We view the blocks of $G$ as forming a partition of the set $\operatorname{Irr}(G)$, and we note that the defect of a block $B$ is the maximum of the defects of the characters in $B$. It is well known that if $B$ contains a character $\chi$ having defect 0 , then $\chi$ is the unique character in $B$, and thus $B$ has defect 0 . Also, by a result of Brauer, if a block $B$ contains a character with defect 1 , then the defect of every character in $B$ is at most 1. (This is Theorem 3 of [2], and we note that the case where $p=2$ is proved separately in Section 8 of Brauer's paper.) Since a block that contains a character having defect 1 cannot also contain a character having defect 0 , we see that if $B$ contains a defect 1 character, then all characters in $B$ have defect 1 , and it follows that $B$ has defect 1 . The union of all blocks of defect 1 , therefore, is exactly the set of irreducible characters having defect 1 .

Associated with each block $B$ of $G$ is a unique conjugacy class of $p$-subgroups of $G$ called the defect groups of $B$, and these subgroups have order $p^{d}$, where $d$ is the defect of $B$. Also, $\mathbf{O}_{p}(G)$ is contained in the defect groups of every block of $G$. (See Corollary 15.39 of [7].)

Brauer's first main theorem asserts that for every $p$-subgroup $Q$ of $G$, there is a bijection from the set of blocks of $G$ for which $Q$ is a defect group onto the set of blocks of $N=\mathbf{N}_{G}(Q)$ for which $Q$ is a defect group. (See, for example, Theorem 15.45 of [7] or Theorem 4.12 of [13.) Thus if $Q$ is a defect of some block of $G$, then $Q$ is also a defect group of some block of $N$, and thus $\mathbf{O}_{p}(N) \subseteq Q$. If $Q$ is a defect group of a block, therefore, it follows that $Q=\mathbf{O}_{p}\left(\mathbf{N}_{G}(Q)\right)$, and thus $Q$ is a radical $p$-subgroup of $G$.

Next, we restate Theorem E(c), which we prove as an application of Theorem 6.2.

Theorem 6.5. Let $G$ have order divisible by $p$, and write $b(G)$ to denote the number of Brauer p-blocks of $G$ that have positive defect. Then the function $b$ is chain local.

Proof. The defect groups of a block $B$ of $G$ form a conjugacy class of radical $p$ subgroups of $G$ having order $p^{d}$, where $d$ is the defect of $B$. This defines a map from 
the set of blocks of $G$ having positive defect to the set of conjugacy classes of nonidentity radical $p$-subgroups of $G$. Writing $f(X, Q)$ to denote the number of blocks of a group $X$ for which $Q$ is a defect group, we thus have $b(G)=\sum_{Q} f(G, Q)$, where $Q$ runs over a set of representatives of the conjugacy classes of nonidentity radical $p$-subgroups of $G$. Also, by Brauer's first main theorem, we see that $f(G, Q)=f(N, Q)$ for each $p$-subgroup $Q$ of $G$, where $N=\mathbf{N}_{G}(Q)$.

Now define the isomorphism-constant function $h$ by letting $h(X)$ be the number of blocks of $X$ for which $\mathbf{O}_{p}(X)$ is a defect group. If $Q$ is a radical subgroup of $G$ and $N=\mathbf{N}_{G}(Q)$, then $Q=\mathbf{O}_{p}(N)$, and thus $h(N)=f(N, Q)=f(G, Q)$. It follows that

$$
b(G)=\sum_{Q} h\left(\mathbf{N}_{G}(Q)\right),
$$

where $Q$ runs over a set of representatives of the conjugacy classes of nonidentity radical $p$-subgroups of $G$. By Theorem 6.2, therefore, the function $b$ is chain local, as required.

By the block-free version of Dade's ordinary conjecture, we expect that all of the functions $k_{d}$ for $d>0$ are chain local, but using some deep results from block theory, we can actually prove that the function $k_{1}$ is chain local. The following is TheoremE(d).

Theorem 6.6. The function $k_{1}$ is chain local, where $k_{1}(G)$ is the number of irreducible characters of $G$ that have defect 1 .

Proof. We have observed that the irreducible characters of $G$ that have defect 1 are exactly the irreducible characters that lie in blocks of defect 1 . Also, the blocks of defect 1 are exactly the blocks that have a subgroup $Q$ of order $p$ as a defect group. Writing $g(Q)$ to denote the number of irreducible characters of $G$ that lie in blocks having defect group $Q$, it follows that

$$
k_{1}(G)=\sum_{Q} g(Q)
$$

where the sum runs over a set a representatives of the $G$-classes of subgroups $Q$ of $G$ having order $p$.

Suppose now that $Q \subseteq G$, where $|Q|=p$, and write $N=\mathbf{N}_{G}(Q)$. By Brauer's first main theorem, there is a bijection $B \mapsto b$ from the set of blocks $B$ of $G$ for which $Q$ is a defect group to the set of blocks $b$ of $N$ for which $Q$ is a defect group. It follows by results of Dade that the numbers of irreducible characters in $B$ and in $b$ are equal. (More precisely, Part 1 of Theorem 1 of $[5]$ describes the number of irreducible characters of $B$, expressed in terms of the group $N$ and the block $b$. Applying this to the block $b$ of $N$, we of course obtain the same answer.)

It follows by Dade's theorem that $g(Q)$ is equal to the number of irreducible characters of $N=\mathbf{N}_{G}(Q)$ that belong to blocks having defect group $Q$. Since $Q \triangleleft N$, we see that $Q$ is contained in the defect groups of each block of $N$, so the blocks of $N$ that have defect group $Q$ are all of the blocks of $N$ that have defect 1. The characters of $N$ that lie in the blocks having defect group $Q$, therefore, are exactly the characters that lie in blocks of defect 1 , and we have seen these are exactly the defect 1 irreducible characters of $N$. It follows that

$$
k_{1}(G)=\sum_{Q} k_{1}\left(\mathbf{N}_{G}(Q)\right),
$$


where, as before, we sum over representatives $Q$ of the conjugacy classes of subgroups of order $p$ in $G$.

Finally, we argue that if $Q$ is a $p$-subgroup with order exceeding $p$, then since $Q$ is contained in every defect group of a block of $\mathbf{N}_{G}(Q)$, no such block can have defect 1 , and thus $k_{1}\left(\mathbf{N}_{G}(Q)\right)=0$. In the above formula for $k_{1}(G)$, therefore, we can sum over a set of representatives of all conjugacy classes of nonidentity $p$-subgroups of $G$, and it follows by Theorem 6.1 that $k_{1}$ is chain local.

It is natural to ask which chain-local functions with values in an abelian group $U$ can be constructed using Theorems 6.1 and 6.2. The answer is "all of them" except that for Theorem 6.1, we must assume that the group $U$ is divisible. (Recall that an abelian group $U$ is divisible if for every element $u \in U$ and every positive integer $t$, there exists a unique element $v \in U$ such that $t v=u$.) Since the additive group of the rational numbers is divisible, we see that the following result includes Theorem G.

Theorem 6.7. Let $f$ be a chain-local function with values in an abelian group $U$.

(a) If $U$ is divisible, there exists a unique $U$-valued isomorphism-constant function $h$ defined on groups $N$ with $\mathbf{O}_{p}(N)>1$ and such that if $p$ divides $|G|$, then $f(G)=\sum_{Q} h\left(\mathbf{N}_{G}(Q)\right)$, where $Q$ runs over a set of representatives of the conjugacy classes of nonidentity p-subgroups of $G$.

(b) With no extra assumption on $U$, there exists a unique $U$-valued isomorphismconstant function $h$ defined on groups $N$ with $\mathbf{O}_{p}(N)>1$ and such that if $p$ divides $|G|$, then $f(G)=\sum_{Q} h\left(\mathbf{N}_{G}(Q)\right)$, where $Q$ runs over a set of representatives of the conjugacy classes of nonidentity radical p-subgroups of $G$.

Proof. Given an arbitrary finite group $N$ with $\mathbf{O}_{p}(N)>1$, we use induction on $|N|$ to define the element $h(N)$ of $U$ and to prove that $h(N)$ is uniquely determined. We assume, therefore, that $h(M)$ has already been defined and that its uniqueness has been established for all groups $M$ with $\mathbf{O}_{p}(M)>1$ and $|M|<|N|$.

To prove (a), let $\mathcal{S}$ be a set of representatives of the conjugacy classes of nonidentity $p$-subgroups $Q$ of $N$ such that $\mathbf{N}_{N}(Q)<N$. Also, let $\mathcal{T}$ be the set of nonidentity normal $p$-subgroups of $N$, and note that $\mathcal{S}$ may be empty, but $|\mathcal{T}|>0$ since by assumption, $\mathbf{O}_{p}(N)>1$.

Now $\mathcal{S} \cup \mathcal{T}$ is a set of representatives of the conjugacy classes of all nonidentity $p$-subgroups $Q$ of $N$, and we require that

$$
\begin{aligned}
f(N)=\sum_{Q \in \mathcal{S} \cup \mathcal{T}} h\left(\mathbf{N}_{N}(Q)\right) & =\sum_{Q \in \mathcal{S}} h\left(\mathbf{N}_{G}(Q)\right)+\sum_{Q \in \mathcal{T}} h\left(\mathbf{N}_{G}(Q)\right) \\
& =\sum_{Q \in \mathcal{S}} h\left(\mathbf{N}_{G}(Q)\right)+|\mathcal{T}| h(N) .
\end{aligned}
$$

Since the sum over $Q \in \mathcal{S}$ is known by the inductive hypothesis, we are forced to define

$$
h(N)=\frac{1}{|\mathcal{T}|}\left(f(N)-\sum_{Q \in \mathcal{S}} h\left(\mathbf{N}_{N}(Q)\right)\right),
$$

where division by the positive integer $|\mathcal{T}|$ makes sense since by assumption, $U$ is a divisible group. 
We see now that $h$ is uniquely defined, and the definition guarantees that the required equation $f(G)=\sum_{Q} h\left(\mathbf{N}_{G}(Q)\right)$ holds for all groups $G$ such that $\mathbf{O}_{p}(G)>1$, where the sum runs over a representative set for the conjugacy classes of nonidentity $p$-subgroups of $G$. To show that this equation holds for all groups $G$ having order divisible by $p$, we define $f_{0}(G)=\sum_{Q} h\left(\mathbf{N}_{G}(Q)\right)$, and we show that $f_{0}=f$.

Now $f$ is chain local by hypothesis, and $f_{0}$ is chain local by Theorem 6.1 , and we know that $f$ and $f_{0}$ agree on groups $G$ such that $\mathbf{O}_{p}(G)>1$. It follows by Lemma 5.5 that $f_{0}(G)=f(G)$ for all groups $G$ having order divisible by $p$, and this completes the proof of (a).

The proof for (b) is similar, but in this case, we take $\mathcal{S}$ to be a set of representatives of the conjugacy classes of nonidentity radical $p$-subgroups $Q$ of $N$ such that $\mathbf{N}_{N}(Q)<N$, and we let $\mathcal{T}$ be the set of nonidentity radical normal $p$-subgroups of $N$. Observe that $\mathcal{T}=\left\{\mathbf{O}_{p}(N)\right\}$, so $|\mathcal{T}|=1$, and in this case, we are forced to define

$$
h(N)=f(N)-\sum_{Q \in \mathcal{S}} h\left(\mathbf{N}_{N}(Q)\right),
$$

and since we have no need to divide by an integer that may exceed 1, we can drop the requirement that the abelian group $U$ is divisible.

The definition of $h$ guarantees that $f(G)=\sum_{Q} h\left(\mathbf{N}_{G}(Q)\right)$ for groups $G$ such that $\mathbf{O}_{p}(G)>1$, where the sum runs over representatives of the conjugacy classes of nonidentity radical $p$-subgroups of $G$. We complete the proof as we did for (a) by defining $f_{0}(G)=\sum_{Q} h\left(\mathbf{N}_{G}(Q)\right)$ and deducing by Lemma 5.5 that $f_{0}=f$. In this case, however, we appeal to Theorem 6.2 to show that $f_{0}$ is chain local.

\section{7. $p$-SOLVABLE GROUPS}

We assume now that the underlying family $\mathcal{F}$ is the family of $p$-solvable groups. Suppose that $f$ is an isomorphism-constant function defined for $p$-solvable groups $G$ having order divisible by $p$, and assume that the values of $f$ lie in some abelian group $U$. Recall that $f$ is chain local for $p$-solvable groups if

$$
\sum_{C \in \mathcal{R}}(-1)^{\operatorname{len}(C)} f\left(G_{C}\right)=0
$$

for all such groups $G$, where as usual, $\mathcal{R}$ is a set of representatives of the $G$-orbits of chains in $G$. Recall also that $f$ is strongly chain local for $p$-solvable groups if for all elements $u \in U$, the functions $f_{(u)}$ are chain local for $p$-solvable groups.

Theorem 7.1. Let $f$ be a $U$-valued isomorphism-constant function defined for $p$ solvable groups $G$ having order divisible by $p$, and suppose that $f(G / L)=f(G)$ whenever $L$ is a normal $p^{\prime}$-group of $G$. Then $f$ is strongly chain local for p-solvable groups.

To prove Theorem 7.1, we need the following preliminary result, which relates the $p$-chains of a group $G$ to the $p$-chains of a factor group $G / L$, where $L$ is a normal $p^{\prime}$-subgroup of $G$. We use the standard "bar convention", so if $L \triangleleft G$ and $\bar{G}=G / L$, then for each subset $X \subseteq G$, we write $\bar{X}$ to denote the image of $X$ under the canonical homomorphism from $G$ onto $G / L$, and in particular, if $x \in G$, then $\bar{x}$ is the coset $L x$. 
Lemma 7.2. Let $L \triangleleft G$, where $L$ is a $p^{\prime}$-group, and write $\bar{G}=G / L$. If $C=\left\{P_{i}\right\}$ is a collection of p-subgroups of $G$, write $\bar{C}=\left\{\overline{P_{i}}\right\}$, so $\bar{C}$ is a collection of p-subgroups of $\bar{G}$. The following then hold:

(a) If $C$ is a chain in $G$, then $\bar{C}$ is a chain in $\bar{G}$, and $\operatorname{len}(\bar{C})=\operatorname{len}(C)$.

(b) Every chain in $\bar{G}$ has the form $\bar{C}$ for some chain $C$ in $G$.

(c) If $B$ and $C$ are chains in $G$ such that $\bar{B}=\bar{C}$, then $B^{x}=C$ for some element $x \in L$.

(d) If $H$ is the stabilizer of a chain $C$ in $G$, then $\bar{H}$ is the stabilizer of $\bar{C}$ in $\bar{G}$.

(e) If $\mathcal{R}$ is a set of representatives of the $G$-orbits of chains in $G$, then $\overline{\mathcal{R}}=$ $\{\bar{C} \mid C \in \mathcal{R}\}$ is a set of representatives of the $\bar{G}$-orbits of chains in $\bar{G}$, and the map $C \mapsto \bar{C}$ is a bijection from $\mathcal{R}$ onto $\overline{\mathcal{R}}$.

Proof. If $C=\left\{P_{i}\right\}$ is a chain, we have $1=P_{0}<P_{1}<\cdots<P_{n}$ and $n=\operatorname{len}(C)$, so to prove (a), it suffices to show that if $P<Q$ are $p$-subgroups of $G$, then $\bar{P}<\bar{Q}$. Clearly, $\bar{P} \subseteq \bar{Q}$, and since $L$ is a $p^{\prime}$-group, we have $|\bar{P}|=|P|<|Q|=|\bar{Q}|$. Then $\bar{P} \neq \bar{Q}$, and thus $\bar{P}<\bar{Q}$, as required.

For (b), consider a chain in $\bar{G}$. Since every subgroup of $\bar{G}$ has the form $\bar{X}$ for some subgroup $X \subseteq G$ with $L \subseteq X$, the given chain in $\bar{G}$ has the form $\left\{\overline{X_{i}}\right\}$, where $L=X_{0}<X_{1}<\cdots<X_{n}$ and the $\overline{X_{i}}$ are $p$-groups. Let $Q$ be a Sylow $p$-subgroup of $X_{n}$, and write $P_{i}=X_{i} \cap Q$, so $1=P_{0} \subseteq P_{1} \subseteq \cdots \subseteq P_{n}=Q$. Now $X_{n}=L Q$ because $X_{n} / L=\overline{X_{n}}$ is a $p$-group, so we have $L \subseteq X_{i} \subseteq X_{n}=L Q$. Dedekind's lemma yields $X_{i}=L\left(X_{i} \cap Q\right)=L P_{i}$, and thus $\overline{X_{i}}=\overline{P_{i}}$ for all $i$. Since the subgroups $\overline{X_{i}}$ are distinct, it follows that the $P_{i}$ must also be distinct, and so $1=P_{0}<P_{1}<\cdots<P_{n}$. Then $C=\left\{P_{i}\right\}$ is a chain, and $\bar{C}=\left\{\overline{P_{i}}\right\}=\left\{\overline{X_{i}}\right\}$, which is the given chain in $\bar{G}$.

To prove (c) we show that up to conjugacy by an element of $L$, each chain $C$ in $G$ is uniquely determined by its image $\bar{C}$. Let $C=\left\{P_{i}\right\}$ where $1=P_{0}<P_{1}<$ $\cdots<P_{n}$, and observe that the full preimage in $G$ of the term $\overline{P_{i}}$ of $\bar{C}$ is $L P_{i}$. The subgroups $L P_{i}$ are thus uniquely determined by $\bar{C}$, and in particular, $L P_{n}$ is uniquely determined by $\bar{C}$. Since $P_{n}$ is a Sylow $p$-subgroup of $L P_{n}$, it follows that up to conjugacy by an element of $L$, the subgroup $P_{n}$ is uniquely determined by $\bar{C}$. We will show that $P_{i}=P_{n} \cap L P_{i}$, and that will imply that $P_{n}$ and $\bar{C}$ together uniquely determine the chain $\left\{P_{i}\right\}=C$, and it will then follow, as required, that $\bar{C}$ determines $C$ up to conjugacy by an element of $L$.

We show now that $P_{i}=P_{n} \cap L P_{i}$. Observe that

$$
P_{i} \subseteq\left(P_{n} \cap L P_{i}\right) \subseteq P_{n} \quad \text { and } \quad P_{i} \subseteq\left(P_{n} \cap L P_{i}\right) \subseteq L P_{i},
$$

so $\left|\left(P_{n} \cap L P_{i}\right): P_{i}\right|$ divides both the $p$-power $\left|P_{n}: P_{i}\right|$ and the $p^{\prime}$-number $\left|L P_{i}: P_{i}\right|$. It follows that $\left|\left(\mid P_{n} \cap L P_{i}\right): P_{i}\right|=1$, so $P_{i}=P_{n} \cap L P_{i}$ as wanted, and this completes the proof of (c).

Next, let $C$ be a chain in $G$ with stabilizer $G_{C}=H$. If $h \in H$, then $(\bar{C})^{\bar{h}}=$ $\overline{C^{h}}=\bar{C}$, so $\bar{H}$ stabilizes $\bar{C}$. To complete the proof of (d), we must show that each element of $\bar{G}$ that stabilizes $\bar{C}$ lies in $\bar{H}$. Suppose, then, that $g \in G$ and $\bar{g}$ stabilizes $\bar{C}$. We have $\bar{C}=(\bar{C})^{\bar{g}}=\overline{C^{g}}$, so we can apply (c) to deduce that $\left(C^{g}\right)^{x}=C$ for some element $x \in L$. Then $g x \in H$, so $\bar{g}=\overline{g x} \in \bar{H}$, as required.

Now, suppose that $\mathcal{R}$ is a set of representatives of the $G$-orbits of chains in $G$. To show that $\overline{\mathcal{R}}$ is a set of representatives of the $\bar{G}$-orbits of chains in $\bar{G}$, we must show that every chain in $\bar{G}$ is $\bar{G}$-conjugate to some member of $\overline{\mathcal{R}}$, and we must also show 
that distinct members of $\overline{\mathcal{R}}$ cannot be conjugate in $\bar{G}$. By (b), an arbitrary chain in $\bar{G}$ has the form $\bar{C}$ for some chain $C$ in $G$, so we must show that $\bar{C}$ is conjugate to a member of $\overline{\mathcal{R}}$. Now $C^{g} \in \mathcal{R}$ for some element $g \in G$, and thus $(\bar{C})^{\bar{g}}=\overline{C^{g}} \in \overline{\mathcal{R}}$, as required.

Now let $B, C \in \mathcal{R}$, so $\bar{B}$ and $\bar{C}$ are typical members of $\overline{\mathcal{R}}$. We will show that if $\bar{B}$ and $\bar{C}$ are conjugate in $\bar{G}$, then $B=C$, and thus $\bar{B}=\bar{C}$. This will complete the proof that $\overline{\mathcal{R}}$ is a set of representatives of the chains of $\bar{G}$, and it will also show that our map $\mathcal{R} \rightarrow \overline{\mathcal{R}}$ is injective, and hence it is a bijection. We suppose, therefore, that $(\bar{B})^{\bar{g}}=\bar{C}$ for some element $g \in G$. Then $\overline{B^{g}}=\bar{C}$, and thus (c) guarantees that $B^{g x}=C$ for some element $x$ in $L$. It follows that $B$ and $C$ are conjugate in $G$, and since we are assuming that $B, C \in \mathcal{R}$, we deduce that $B=C$. This completes the proof of $(\mathrm{e})$.

Proof of Theorem 7.1. Let $G$ be an arbitrary $p$-solvable group having order divisible by $p$, and let $\mathcal{R}$ be a set of representatives of the $G$-orbits of chains in $G$. Also, let $L=\mathbf{O}_{p^{\prime}}(G)$, and write $\bar{G}=G / L$, so $\mathbf{O}_{p}(\bar{G})>1$.

Let $\alpha$ be the conjugacy-constant function on $\bar{G}$ obtained by restricting $f$ to the subgroups of $\bar{G}$. Corollary 4.2 guarantees that $\alpha$ is chain local on $\bar{G}$, and since we know by Lemma 7.2 (e) that $\overline{\mathcal{R}}$ is a set of representatives of the $\bar{G}$-orbits of chains of $\bar{G}$, we have

$$
0=\sum_{\bar{C} \in \overline{\mathcal{R}}}(-1)^{\operatorname{len}(\bar{C})} \alpha\left(\bar{G}_{\bar{C}}\right)=\sum_{C \in \mathcal{R}}(-1)^{\operatorname{len}(C)} f\left(\overline{G_{C}}\right)=\sum_{C \in \mathcal{R}}(-1)^{\operatorname{len}(C)} f\left(G_{C}\right) .
$$

Here, to obtain the second equality, we observe that $\alpha$ agrees with $f$ on subgroups of $\bar{G}$, and we apply several of the conclusions of Lemma 7.2. Specifically, we use the equalities $\operatorname{len}(\bar{C})=\operatorname{len}(C)$ and $\bar{G}_{C}=\bar{G}_{\bar{C}}$, and we use the fact that the map $C \mapsto \bar{C}$ defines a bijection from $\mathcal{R}$ onto $\overline{\mathcal{R}}$. The third equality above holds by the hypothesis on $f$, which applies because $\overline{G_{C}} \cong G_{C} /\left(G_{C} \cap L\right)$ and $G_{C} \cap L$ is a normal $p^{\prime}$-subgroup of $G_{C}$.

Since $G$ is an arbitrary $p$-solvable group having order divisible by $p$, and we have just seen that the chain sum on $G$ with respect to $f$ vanishes, it follows that $f$ is chain local for $p$-solvable groups.

To complete the proof, we must show that each of the functions $f_{(u)}$ for $u \in U$ is chain local for $p$-solvable groups. By the first part of the proof, it suffices to show for all $u \in U$ that $f_{(u)}(G / L)=f_{(u)}(G)$ whenever $L$ is a normal $p^{\prime}$-subgroup $G$. To see this, let $f(G)=v$, where $v \in U$. Then $f(G / L)=v$, so if $v=u$, we have $f_{(u)}(G)=1=f_{(u)}(G / L)$, and otherwise, $f_{(u)}(G)=0=f_{(u)}(G / L)$.

Recall now that by Theorem $\mathrm{E}(\mathrm{a})$, the function that counts the conjugacy classes of $p$-elements in a group $G$ is chain local. Experiment shows that in general, this function is not strongly chain local, but nevertheless, we have the following.

Corollary 7.3. Let $f$ be the isomorphism-constant function defined by letting $f(G)$ be the number of conjugacy classes consisting of p-elements in a group $G$. Then the function $f$ is strongly chain local for p-solvable groups.

Proof. By Theorem 7.1, it suffices to show that if $G$ is $p$-solvable and $L \triangleleft G$ is a $p^{\prime}$-subgroup, then $f(G)=f(\bar{G})$, where $\bar{G}=G / L$. Since this is fairly well known, we sketch the proof, omitting some details.

It is easy to see that the map $x \mapsto \bar{x}$ carries the set of $p$-elements of $G$ onto the set of $p$-elements of $\bar{G}$, and it is clear that if $x$ and $y$ are conjugate elements of $G$, 
then $\bar{x}$ and $\bar{y}$ are conjugate in $\bar{G}$. The map $x \mapsto \bar{x}$ thus induces a surjective map from the set of classes of $p$-elements of $G$ onto the set of classes of $p$-elements of $\bar{G}$, so to prove that $f(G)=f(\bar{G})$, it suffices to show that this map is injective.

If $x$ and $y$ are $p$-elements of $G$ and $\bar{x}$ and $\bar{y}$ are conjugate in $\bar{G}$, we must show that $x$ and $y$ are conjugate in $G$. Replacing $x$ by a conjugate, we can assume that $\bar{x}=\bar{y}$, and thus $L\langle x\rangle=L\langle y\rangle$. Now $\langle x\rangle$ and $\langle y\rangle$ are Sylow $p$-subgroups of $L\langle x\rangle$, so replacing $x$ by a conjugate once again, we can assume that $\langle x\rangle=\langle y\rangle$. Then $x y^{-1} \in L \cap\langle x\rangle=1$, so $x=y$, and the proof is complete.

Our final two results concern $p$-special and $p^{\prime}$-special characters of $p$-solvable groups, and we refer the reader to [9] for the definitions and basic properties of these objects.

Corollary 7.4. Let $f$ be the isomorphism-constant function defined by letting $f(G)$ be the number of p-special irreducible characters of a p-solvable group $G$. Then $f$ is strongly chain local for p-solvable groups.

Proof. Let $L \triangleleft G$ and write $\bar{G}=G / L$. It is well known that there is a natural bijection $\psi \mapsto \tilde{\psi}$ from $\operatorname{Irr}(\bar{G})$ onto $\{\chi \in \operatorname{Irr}(G) \mid L \subseteq \operatorname{ker}(\chi)\}$, where by definition, $\tilde{\psi}(g)=\psi(\bar{g})$.

Suppose now that $G$ is $p$-solvable. Given $\psi \in \operatorname{Irr}(\bar{G})$, it is not hard to see that $\tilde{\psi}$ is $p$-special if and only if $\psi$ is $p$-special. Also, if $L$ is a $p^{\prime}$-group, then for all $p$-special characters $\chi$ of $G$, we have $L \subseteq \operatorname{ker}(\chi)$, and thus $\chi=\tilde{\psi}$ for some character $\psi \in \operatorname{Irr}(\bar{G})$, and we see that $\psi$ must be $p$-special. It follows that if $L$ is a $p^{\prime}$-group, then the map $\psi \mapsto \tilde{\psi}$ defines a bijection from the set of $p$-special characters of $\bar{G}$ onto the set of $p$-special characters of $G$, and thus $f(\bar{G})=f(G)$. We conclude by Theorem 7.1 that $f$ is strongly chain local, as required.

The corresponding result for $p^{\prime}$-special characters is true, but the only proof that we see relies on the following relatively deep result, which is a consequence of a theorem of Isaacs, proved by Wolf in [19].

Corollary 7.5. Let $f$ be the isomorphism-constant function defined for p-solvable groups $G$ by letting $f(G)$ be the number of $p^{\prime}$-special irreducible characters of $G$. Then $f(G)=f(N)$, where $N$ is the normalizer in $G$ of a Sylow p-subgroup.

Proof. Given a $p$-solvable group $G$, it is a consequence of Corollary 1.16 of [19] that $f(G)=k(H)$, where $H$ is a Hall $p^{\prime}$-subgroup of $N$, and as usual, $k$ is the function that counts conjugacy classes. Applying this to the group $N$, we see that also $f(N)=f(H)$, and so $f(G)=f(N)$.

Corollary 7.6. Let $f$ be the isomorphism-constant function defined for p-solvable groups by letting $f(G)$ be the number of $p^{\prime}$-special irreducible characters of $G$. Then $f$ is strongly chain local for p-solvable groups.

Proof. Corollary 7.6 guarantees that the hypothesis of Corollary [5.3] is satisfied, and thus $f$ is strongly chain local for $p$-solvable groups.

\section{ACKNowledgments}

Both authors thank G. Malle and the referee for a careful reading of the manuscript. 


\section{REFERENCES}

[1] J. L. Alperin, Weights for finite groups, The Arcata Conference on Representations of Finite Groups (Arcata, Calif., 1986), Proc. Sympos. Pure Math., vol. 47, Amer. Math. Soc., Providence, RI, 1987, pp. 369-379. MR933373

[2] Richard Brauer, Investigations on group characters, Ann. of Math. (2) 42 (1941), 936-958, DOI 10.2307/1968775. MR5731

[3] Kenneth S. Brown, Euler characteristics of groups: the p-fractional part, Invent. Math. 29 (1975), no. 1, 1-5, DOI 10.1007/BF01405170. MR385008

[4] Kenneth S. Brown, High dimensional cohomology of discrete groups, Proc. Nat. Acad. Sci. U.S.A. 73 (1976), no. 6, 1795-1797, DOI 10.1073/pnas.73.6.1795. MR419682

[5] E. C. Dade, Blocks with cyclic defect groups, Ann. of Math. (2) 84 (1966), 20-48, DOI 10.2307/1970529. MR200355

[6] Everett C. Dade, Counting characters in blocks. I, Invent. Math. 109 (1992), no. 1, 187-210, DOI 10.1007/BF01232023. MR1168370

[7] I. Martin Isaacs, Character theory of finite groups, Dover Publications, Inc., New York, 1994. Corrected reprint of the 1976 original [Academic Press, New York; MR0460423 (57 \#417)]. MR:1280461

[8] I. Martin Isaacs, Finite group theory, Graduate Studies in Mathematics, vol. 92, American Mathematical Society, Providence, RI, 2008. MR2426855

[9] I. Martin Isaacs, Characters of solvable groups, Graduate Studies in Mathematics, vol. 189, American Mathematical Society, Providence, RI, 2018. MR3791517

[10] Radha Kessar and Markus Linckelmann, Dade's ordinary conjecture implies the AlperinMcKay conjecture, Arch. Math. (Basel) 112 (2019), no. 1, 19-25, DOI 10.1007/s00013-0181230-9. MR 3901898

[11] Reinhard Knörr and Geoffrey R. Robinson, Some remarks on a conjecture of Alperin, J. London Math. Soc. (2) 39 (1989), no. 1, 48-60, DOI 10.1112/jlms/s2-39.1.48. MR989918

[12] Burkhard Külshammer and Geoffrey R. Robinson, On Alperin's conjecture and certain subgroup complexes, Algebr. Represent. Theory 1 (1998), no. 4, 383-398, DOI 10.1023/A:1009961408896. MR 1683619

[13] G. Navarro, Characters and blocks of finite groups, London Mathematical Society Lecture Note Series, vol. 250, Cambridge University Press, Cambridge, 1998. MR 1632299

[14] Gabriel Navarro, Character theory and the McKay conjecture, Cambridge Studies in Advanced Mathematics, vol. 175, Cambridge University Press, Cambridge, 2018. MR 3753712

[15] Daniel Quillen, Homotopy properties of the poset of nontrivial p-subgroups of a group, Adv. in Math. 28 (1978), no. 2, 101-128, DOI 10.1016/0001-8708(78)90058-0. MR493916

[16] Geoffrey R. Robinson, Local structure, vertices and Alperin's conjecture, Proc. London Math. Soc. (3) 72 (1996), no. 2, 312-330, DOI 10.1112/plms/s3-72.2.312. MR.1367081

[17] Jacques Thévenaz, Locally determined functions and Alperin's conjecture, J. London Math. Soc. (2) 45 (1992), no. 3, 446-468, DOI 10.1112/jlms/s2-45.3.446. MR.1180255

[18] P. J. Webb, Subgroup complexes, The Arcata Conference on Representations of Finite Groups (Arcata, Calif., 1986), Proc. Sympos. Pure Math., vol. 47, Amer. Math. Soc., Providence, RI, 1987, pp. 349-365. MR.933372

[19] Thomas R. Wolf, Variations on McKay's character degree conjecture, J. Algebra 135 (1990), no. 1, 123-138, DOI 10.1016/0021-8693(90)90153-F. MR.1076081

Department of Mathematics, University of Wisconsin, 480 Lincoln Drive, Madison, WisCONSIN 53706

Email address: isaacs@math.wisc.edu

Departament de Matemàtiques, Universitat de València, 46100 Burjassot. València, SPAIN

Email address: gabriel@uv.es 